\title{
Survey on advances on terrain based navigation for autonomous underwater vehicles
}

\author{
José Melo ${ }^{\mathrm{a}, \mathrm{b}, *}$, Aníbal Matos ${ }^{\mathrm{a}, \mathrm{b}}$ \\ a FEUP - Faculty of Engineering, University of Porto, Rua Dr. Roberto Frias s/n, 4200-465 Porto, Portugal \\ b INESC TEC - INESC Technology and Science, Rua Dr. Roberto Frias 378, 4200-465 Porto, Portugal
}

\section{A R T I C L E I N F O}

\section{Keywords:}

AUV navigation

AUV autonomy

Terrain based navigation for AUVs

\begin{abstract}
A B S T R A C T
The autonomy of robotic underwater vehicles is dependent on the ability to perform long-term and long-range missions without need of human intervention. While current state-of-the-art underwater navigation techniques are able to provide sufficient levels of precision in positioning, they require the use of support vessels or acoustic beacons. This can pose limitations on the size of the survey area, but also on the whole cost of the operations.

Terrain Based Navigation is a sensor-based navigation technique that bounds the error growth of deadreckoning using a map with terrain information, provided that there is enough terrain variability. An obvious advantage of Terrain Based Navigation is the fact that no external aiding signals or devices are required. Because of this unique feature, terrain navigation has the potential to dramatically improve the autonomy of Autonomous Underwater Vehicles (AUVs).

This paper consists on a comprehensive survey on the recent developments for Terrain Based Navigation methods proposed for AUVs. The survey includes a brief introduction to the original Terrain Based Navigation formulations, as well as a description of the algorithms, and a list of the different implementation alternatives found in the literature. Additionally, and due to the relevance, Bathymetric SLAM techniques will also be discussed.
\end{abstract}

\section{Introduction}

Navigation is a broad and extensive field of study, and one of the key issues in modern robotics. In a general sense Navigation encompasses all the sub-problems related to moving a robot from one point to another, thus including tasks like robot self localization, trajectory planning, collision avoidance or mapping, among others. Notwithstanding, for the Marine Robotics community the term Navigation is loosely applied to self-localization tasks only, and that is the understanding that will be followed throughout the remainder of this article.

According to Hagen et al. (2009), the level of autonomy achieved by Autonomous Underwater Vehicles (AUVs) is chiefly determined by their performance in three areas, namely energy autonomy, navigation autonomy and decision autonomy. Navigation autonomy is then described as the ability to navigate precisely and obtain positioning solutions with little or non-significant errors over extended periods of time. Therefore, the level of autonomy of robotic underwater vehicles is dependent on the ability to perform long-term and long-range missions with little or no human intervention. This is of particular importance for autonomous operation in deep water or covert military operations, that require the vehicles to handle submerged operation for long periods of time, and covering long ranges. Enabling such missions is therefore a challenging goal for the AUV community, and one that would dramatically improve the level of autonomy of these vehicles.

The ability autonomous vehicles must have to correctly understand their position and attitude within the environment is determinant for the success of different applications. For field robotics, the Global Navigation Satellite Systems (GNSS), like the American operated Global Positioning System (GPS), have become an invaluable asset to resolve the global position of a given robot. In fact, modern GPS techniques can provide the global position in terms of latitude and longitude with centimetre accuracy, which is considered to be adequate to the vast majority of applications. However, underwater the use of GNSS technology is not possible due to the strong attenuation that affects electromagnetic radiations in aquatic environments.

Terrain Based Navigation, Terrain Relative Navigation or Terrain Aided Navigation are all interchangeable terms used to describe a family of position fixing systems based on the profile of the terrain. Underwater Terrain Based Navigation (TBN), to which research efforts

\footnotetext{
* Corresponding author at: FEUP - Faculty of Engineering, University of Porto, Rua Dr. Roberto Frias s/n, 4200-465 Porto, Portugal

E-mail addresses: jose.melo@fe.up.pt (J. Melo), anibal@fe.up.pt (A. Matos).
} 
have been recently focused, is a potentially powerful solution for longrange navigation of AUVs. Similarly to the use of GPS or even Acoustic Navigation, TBN uses information of the variations of the terrain to bound the errors of inertial navigation, thus increasing the long term estimation accuracy of the position of the vehicle. TBN is a selfcontained technique, in the sense that no external aiding signals or devices are needed. This is in fact a great advantage when comparing to traditional approaches. Because of this unique feature, TBN has the potential to dramatically improve the autonomy of AUVs, given that terrain maps of the areas to navigate are available, and that sufficient terrain variability exists in order to make the terrain informative.

This article aims to be an in-depth survey of existing TBN algorithms for underwater vehicles. However, we are exclusively interested on bathymetry-based, featureless approaches. Even though some authors have proposed vision-based underwater navigation algorithms, the use of rich imaging sensors, like cameras, is generally precluded. Underwater images are known to be degraded due to different environmental factors such as light attenuation, turbidity or floating particles, and only in very close-range applications can cameras be relevant, for example in docking operations. Some sonar sensors can provide acoustic images of the bottom, and various examples of the image-based problem for TBN have been proposed, for example by Strauss et al. (1999); Zerr et al. (2005); Stalder et al. (2008), or Woock and Frey (2010). Such methods, with roots on the image-processing domain, are dependent on feature and landmark matching. With the sea bottom being largely featureless, the use of such techniques is restricted to only a small subset of underwater regions, and mostly to structured environments, as segmentation and classification of natural underwater features is not a straightforward task. For those reasons, this survey is exclusively focused on bathymetric TBN, and image-based TBN won't be further discussed.

By providing a complete overview on all the different aspects, covering from the basic concepts, to sensors used and implementation details, this article also complements previous existing surveys, as the one published by Carreno et al. (2010). Naturally, a special focus is given to the works in the area with a major impact on the AUV community. The article is organized in the following way. Section 2 provides an overview on general methods for underwater navigation, and motivates for use of TBN. On Section 3 an introduction to the general TBN problem is provided, and the section that follows is devoted to deliver an insight on the different sonar sensors that can be used to capture the characteristics of the sea bottom. Section 5 discusses the underwater TBN algorithms while Section 6 presents a discussion on the different state-space models used in the literature. Section 7 introduces the Bayesian Estimation framework and the possible realizations of the general Bayes Filter applied to the specific problem of TBN. Section 8 introduces relevant related work, based on SLAM techniques and scan matching, and finally Section 9 summarizes the key findings.

\section{Underwater navigation}

Underwater navigation can be roughly subdivided in three main branches: Inertial Navigation, Acoustic Navigation, and Geophysical Navigation, the most recent branch. While these different techniques can be used as standalone, they are usually combined into more robust navigation solutions, designed to capture the strengths of each individual method. Up to recently, the state-of-the-art approach has been combining acoustic and inertial navigation systems. Inertial Navigation is a self-contained navigation technique with a very good short-term accuracy, but its position and velocity estimates are known to drift over time. On the other hand, beacon-based Acoustic Navigation techniques are able to provide navigational aids, usually in form of range and bearing signals, but they usually have a lowupdate rate. However, the need for deployment of acoustic beacons or a support vessel is extremely inconvenient, and even impracticable for
Table 1

Summary of advantages and disadvantages of the different underwater navigation techniques.

\begin{tabular}{lll}
\hline & Advantages & Disadvantages \\
\hline Inertial Navigation & Self-contained & Drifts over time \\
Acoustic Navigation & Robust position & Low update rate, deployment of \\
& fixing & external devices \\
Geophysical & Self-contained & Scarcity of maps \\
Navigation & & \\
\hline
\end{tabular}

some applications. While resurfacing and using a GPS receiver can partially solve for this problem, such behaviour is sometimes undesirable, like during deep water surveys, or even be impossible if, for example, the vehicle is navigating under an iced surface. The challenge is then to provide methods that allow AUVs to operate autonomously in highly unstructured environments, and combining Inertial Navigation with Geophysical Navigation methods, might be the way to go.

The advantages and disadvantages of the different underwater navigation techniques have been summarized in Table 1. For reference, in the remainder of this section we provide a brief overview on the existing navigation techniques for AUVs. A more comprehensive survey, covering both the technology and the algorithms, was provided at different times by different authors, as for example Kinsey et al. (2006); Stutters et al. (2008) and more recently Paull et al. (2014).

\subsection{Inertial navigation}

Inertial Navigation is, as the name indicates, based on inertial principles and uses measurements from Inertial Measurement Units (IMUs) to obtain estimates of both position and velocity using deadreckoning techniques. Dead-reckoning is the process of recurrently estimating a navigation solution of a vehicle using a previously known position and orientation, and integrating the vehicle's velocity and acceleration.

Modern IMUs consist on a set of accelerometers and gyroscopes, that measure the specific force and angular velocity, respectively. By using a triad of each of these sensors, mounted along the different coordinated axis, it is possible to obtain by integration three-dimensional position and attitude estimates, with good short time accuracy and high update rates. Sometimes IMUs also contain a triad of magnetometers, to measure Earth's magnetic field, which allows to obtain heading estimates. In alternative, heading estimates can also be obtained by gyrocompassing, provided that the precision of the gyroscopes is high enough to measure the earth's rotation.

The fact that Inertial Navigation is self-contained, in the sense that it neither emits nor receives any external signal, is one of its most significant strengths, making it a stealthy navigation solution, immune to interference or jamming. However, due to the dead-reckoning nature of the process, the navigation errors obtained with this method are known to increase and grow unbounded with time, in an extent that is heavily dependent on the accuracy of the sensors used. This is caused by the noise and bias levels present on the signals coming from the IMUs, which are then continuously integrated. According to Groves (2013), inertial sensors can be roughly divided in 4 categories with respect to their levels of maximum horizontal position drifts, as specified on Table 2.

While with marine and navigation grade IMUs it is possible to have AUVs to perform short-term missions with negligible navigation errors, IMUs in these classes are characterized for having dimensions, cost and power requirements that are not compatible the majority of the up-todate autonomous vehicles. On the other side, for the IMUs in the lowest class, the automotive class, obtaining position estimates only from inertial measurements is hardly possible, this class of low-cost sensors are employed on Attitude and Heading Reference System (AHRS). For IMUs of the remaining classes, or for longer missions, the use of 
Table 2

Inertial Navigation Systems performance categories.

\begin{tabular}{lll}
\hline Grade & Position drift & Application \\
\hline Marine & $<1.8 \mathrm{~km} /$ day & submarine, spacecraft \\
Navigation & $\sim 1.5 \mathrm{~km} /$ hour & airliner, military aircraft \\
Tactical & $>15 \mathrm{~km} /$ hour & guided weapons, UAVs \\
Automotive & n.a. & AHRS, ABS \\
\hline
\end{tabular}

navigational aids are therefore required.

At the core of Inertial Navigation are the Inertial Navigation Systems (INS). These systems are responsible to solve the standard inertial navigation equations of the vehicle, using data coming from the IMU. In addition, Modern INS are also responsible for data fusion of inertial data with position and velocity data, acquired by external sensors. In the case of underwater vehicles, sensors like Doppler Velocity Loggers (DVL) or barometric depth sensors are usually employed. It is also common to obtain external positional aids using any of the acoustic navigation schemes, detailed on the following subsection.

\subsection{Acoustic navigation}

Acoustic Navigation embraces a number of techniques that rely on the exchange of acoustic signals between a set of acoustic beacons, and one or more vehicles, with the objective of determining the position of the latter. Broadly speaking, three distinct Acoustic Navigation schemes exist, namely the Long Baseline (LBL), the Short Baseline (SBL), and the Ultra Short Baseline (USBL). If appropriate acoustic interrogation protocols are used, all of these systems can be used for external tracking of the vehicle, but also to provide relative navigational aids to the vehicles. A detailed review of the different Acoustic Navigation schemes, their individual strengths and their disadvantages has been provided by ,.

SBL and USBL are two acoustic positioning systems that provide relative range and bearing to an acoustic beacon. Differently from those, LBL systems only provide ranges to an array of acoustic beacons, but position estimates can be obtained by applying multilateration techniques. Nevertheless, the ranges provided by these methods are always obtained using the Time-of-Flight of the acoustic signals, and highly dependent on knowing with a high degree of accuracy the local sound wave propagation velocity. Computing bearing, however is done differently for SBL and USBL. On SBL systems, bearing is derived from the time differences of arrival (TDOA), as a ping (transmitted acoustic signal) is detected on each of the transceivers. On the other hand, due to its relatively small baseline, USBL systems compute bearing by comparing the phase of a given ping between individual elements of an acoustic array.

The length of the baseline among such systems differs significantly. as indicated in Table 3. SBL is usually characterized for having the transducers mounted on the hull of the vehicle, as distant from each other as possible. On the other hand USBL systems have the transducers mounted closely to each other. Differently from the previous methods, LBL systems only provide ranges to an array of acoustic beacons deployed on the seafloor, in predefined locations within the operation area. While usually four beacons need to be deployed prior to the operation, in some specific configurations the number of beacons

Table 3

Baseline length for the different types of Acoustic Navigation systems.

\begin{tabular}{ll}
\hline Acoustic System & Baseline Length \\
\hline LBL & $>100 \mathrm{~m}$ to $\sim<2000 \mathrm{~m}$ \\
SBL & $\sim 20 \mathrm{~m}$ to $50 \mathrm{~m}$ \\
USBL & $<10 \mathrm{~cm}$ \\
\hline
\end{tabular}

can be smaller. In those cases some ambiguity with respect to threedimensional position of the vehicle might arise. Loosely speaking, SBL and USBL have a lower level of complexity and don't require the deployment of any additional transponders, but they need to go through a detailed calibration process to obtain optimal precisions on positioning.

One of the main advantages of LBL systems is its very good position accuracy, which is independent of the operational depths. Maybe for that reason, LBL is in fact the most popular of all the acoustic positioning systems. However, the cost and time needed to set up a network, and the later recovery of the beacons, which can be quite cumbersome for adverse environments, is still a big drawback for the use of such systems. To overcome the need of deploying the beacons on the seafloor, the use of GNSS enabled buoys has been proposed, in what can be called Inverted LBL. With the use of such systems, the transponders of the bottom are replaced by floating buoys which carry the acoustic transducers. Due to the fact that such devices also carry GNSS receivers, calibration of the system can be significantly simplified.

Both traditional LBL or the Inverted LBL require the use of multiple acoustic beacons, deployed prior to operations whether on the sea bottom, or on the surface. In order to reduce the logistics associated to LBL, different researchers have focused on developing methods that use only a single acoustic beacon. To name only a few, Larsen (2000) proposed the Synthetic LBL method, which combined dead-reckoning with range measurements from a single acoustic source to provide submeter positioning accuracy. Later on, LaPointe (2006) presented the Virtual LBL system, a single beacon navigation system that uses a virtual network of multiple acoustic transponders that allows an underwater vehicle to navigate similarly to what happens with traditional LBL. The position of each virtual transponders at any given time is then obtained by combining multiple range measurements from the real single transponder and the dead reckoning track of the vehicle. More recently, the work by Ferreira and Matos (2010) simultaneously addressed the problems of localization and control of an AUV using a single acoustic beacon for homing scenarios.

Recent advances in underwater communication topics have also brought Acoustic Modems to play a relevant part in underwater navigation capabilities. Several authors have proposed different frameworks to enable Cooperative Navigation, for example Bahr et al. (2009); Fallon et al. (2010). In Cooperative Navigation, teams of AUVs localize themselves more accurately by sharing position estimates and uncertainty. However, such approaches require a data link between the vehicles. While this is an interesting approach, acoustic communications are still characterized by small bandwidth, low data rates and high latency and, particularly for shallow waters and adverse environmental conditions, reliable underwater communications can be quite challenging for long distances.

\subsection{Geophysical navigation}

Geophysical Navigation uses physical features of the environment where the AUVs navigate to produce an estimate of the location of the vehicle. As stated by Leonard et al. (1998), at its basis this kind of navigation relies on matching sensor data with an a priori environment map, under the assumption that there is sufficient spatial variation in the parameters being measured to permit accurate localization. According to Meduna (2011), perhaps the biggest advantage of these map-based navigation methods is the fact that it is a completely onboard navigation, without the need for external devices, granting the vehicles a large operational range. TBN, the focus of this article that will be thoroughly discussed over the following sections, fits in this category. However other techniques also exist.

Geomagnetic Based Navigation is in fact a related and very similar problem to TBN, with the main difference being the features present in the pre-surveyed digital map. While for TBN the map represents 
terrain elevation, in geomagnetic navigation a Marine Geomagnetism Map is used. Marine Geomagnetism has eight elements, namely geomagnetic total intensity, geomagnetic horizontal component, geomagnetic vertical component, geomagnetic declination, geomagnetic inclination and geomagnetic components in $X, Y$, and $Z$. Comparing with TBN, which uses sonar, Geomagnetic Navigation relies only on magnetic sensors, which are passive sensors. This is an obvious advantage particularly for stealthy operations. Given the similarity between Terrain Based Navigation and Geomagnetic Navigation, all the matching techniques could, in principle, be applied to the latter, up to some minor differences. Different authors have addressed the application of Geomagnetic Navigation to underwater environments, namely by Mu et al. (2007); Ren et al. (2008); Zhao et al. (2009) or Wang et al. (2010). However, up to now conclusive experimental results are still to be presented.

\section{Terrain based navigation}

The goal of TBN is to obtain a position estimate that best fits a set of acquired terrain measurements. This is done by comparing the obtained terrain profile with a prior map or terrain database. In the remainder of this section a description of the seminal work that lead to modern TBN algorithms will be provided, namely the instrumental TERCOM and SITAN methods.

The pioneering techniques for TBN were initially derived to be used on missiles and aerial military vehicles. In an era when the ubiquity of GPS was still not a reality, TBN presented some unique characteristics that lead to its widespread acceptance. The autonomy and robustness against interference or jamming, together with the fact that such systems could be used under all weather conditions, during day or night, where among the main advantages of such algorithms. The ever increasing availability of high-resolution digital terrain maps delivered with the aid of satellites played a great contribution to the widely acceptance of this technique.

Initial techniques eventually evolved into commercial products and nowadays several NATO aircraft are equipped with such systems. Besides providing accurate navigation solutions, modern TBN systems have been extended for both safety and tactical benefits of the aircraft, offering features like predictive ground collision avoidance or obstacle warning, making it particularly suited to military vehicles. A recent description of a commercial TBN system for aircraft has been done by Cowie et al. (2008). More recently, TBN has also been proposed to be used on several space exploration missions, namely for planetary entry, descent and landing by Alexander et al. (2012) and Johnson and Montgomery (2008). For a recent review on the different TBN solutions for aerial vehicles, refer to works by Karabork (2010); Vaman (2012) and the references therein.

Early classifications divided TBN methods on two different broad categories: Batch Methods and Sequential Methods. For reasons that will be made clear ahead, these designations arose due to the nature of TERCOM and SITAN methods, arguably the most influential in the field of terrain navigation. The main differences between these two algorithms are schematically depicted in Fig. 1. Other alternatives were also proposed that were conceptually different from those two meth- ods. However, for various reasons they were never widely adopted by the community. Examples of this are VATAN algorithm, proposed by Enns and Morrell (1995), which uses the Viterbi algorithm applied to the TBN. Recently, Kedong et al. (2006) suggested applying the Iterative Closest Point (ICP) algorithms to the problem of TBN. Pattern matching approaches, using image processing techniques for landmark matching were also proposed, for example by Lucido et al. (1998); Strauss et al. (1999) and others, but they will not be further discussed in this article. Finally some hybrid approaches, combining batch and sequential methods, have arisen most notably TERPROM. Bergman et al. (1999) presented such hybrid solution, that used a correlation based batch method on an initial phase, to obtain a coarse position estimate, and once this was done it used a sequential approach to track the position, using Kalman Filter techniques.

\subsection{TERCOM}

The Terrain Contour-Matching (TERCOM), the first Terrain Based Navigation method ever developed, dates back to the 1950's, when the concept was initially proposed. However, only almost two decades later was the TERCOM system successfully applied to cruise missile guidance systems. According to Vaman (2012), on its original formulation TERCOM was designed to provide positional fixes to update the INS of different vehicles, like aircraft or land-attack cruise missiles. In this method, the positional fix is obtained by taking the best match from a correlation-like function that assesses how well the obtained terrain profile matches an existing map of the terrain. For aerial vehicles terrain profiles are traditionally obtained by combining the readings of barometric altimeters together with laser or radar altimeters.

Even though a navigation Kalman Filter is used, TERCOM can be considered basically a correlation method in the sense that the position fix, which is obtained by matching the sensor-based profile with a bathymetric map, is the measurement input to be used by the navigation filter. Jalving et al. (2001) noted that the term "correlation" can be misleading in the sense that these methods do not compute correlation in a strict statistical sense, but use instead some other matching function that shows how well a measured bottom depth profile matches a map. Usually the Absolute Square Distance (1) or variations of it are used.

$\operatorname{ASD}\left(x_{t}\right)=\sum_{i=1}^{N}\left(z_{t, i}-h\left(x_{t}\right)\right)^{2}$

On the original TERCOM formulation, profiles of the terrain were acquired by collecting a set of readings from the altimeter sensors, which were then simultaneously processed. Because of that, these TBN methods were classified as batch estimation methods. However, as reported by Meduna (2011), due to the fact that the motion of the vehicle between consecutive measurements is not taken into account, these systems are only successful when used with a highly accurate INS or with a dense range sensor. Another limitation of the original TERCOM formulation is the fact that no statistically justified measure of uncertainty in position estimates is provided. A schematic view of
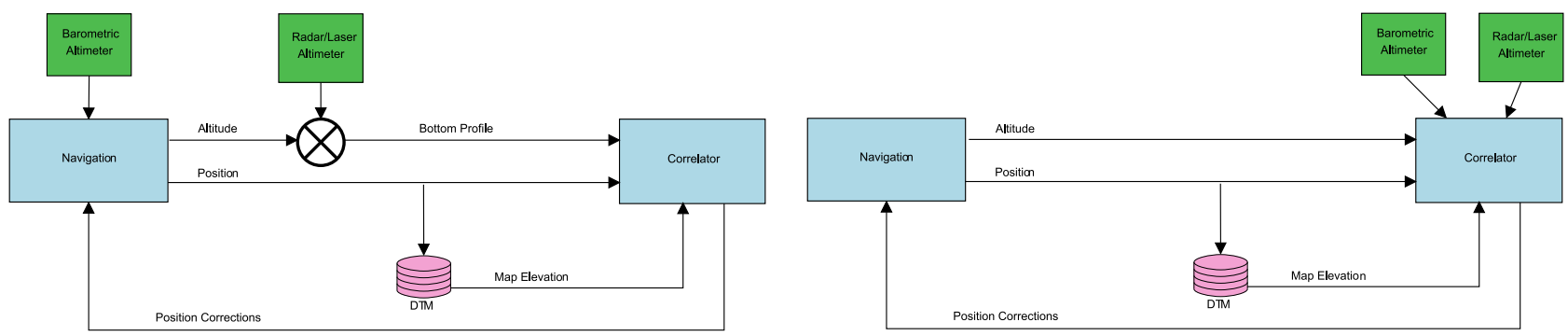

Fig. 1. Schematic view of the two instrumental Terrain Based Navigation methods: TERCOM (1) and SITAN (1). 
the TERCOM method can be found in Fig. 1(a).

\subsection{SITAN}

The Sandia Inertial Terrain Aided Navigation, best known as SITAN, is another fundamental TBN method developed at the Sandia National Labs and first proposed by Hostetler (1978). SITAN, a Kalman Filter based TBN algorithm, was the first sequential method developed, which radically differed from the existing batch methods.

Recalling from the previous subsection, TERCOM uses only the best match from the correlation obtained between a set of terrain measurements, that constitute a terrain profile, and a digital map of the terrain. This best match is then used to update the navigation filter. Opposed to that, the sequential nature of SITAN comes from the fact that every new terrain measurement is processed independently, and used as an input to the navigation filter. A schematic view of the SITAN method can be found in Fig. 1(b). SITAN uses the obtained terrain measurements directly as an update of the navigation filter, which introduces a non-linearity on the measurement equation. Due to its undulating nature, terrain measurements are in fact a nonlinear function of the state of the navigation system. This is can be handled by linearising the TBN measurement equation around the best estimate of the position of the vehicle, an usual procedure when resorting to the Extended Kalman Filter.

SITAN's simplicity, when compared to the computational load required by TERCOM, is one of its the main advantages. Nevertheless, there are two key limitations which are related with the aforementioned linearisation. Because terrain gradients need to be computed, with respect to the latitude, longitude and height components INS position error, the quality of it will have a significant impact on the produced position estimates. This is of particular importance if we consider that natural terrain is highly non-linear. This can be in part addressed by using stochastic linearisation methods as suggested by Hostetler (1978) and Hollowell (1990), or even Multiple Model Adaptive Estimation (MMAE) techniques as proposed by Hostetler and Andreas (1983). Another limitation with this method is related with the fact that gradient are computed at the position estimated by the navigation filter, but deviations from the actual correct position can occur. For this reason, SITAN algorithms always require small initial position errors Fig. 2.

\section{Terrain measurements}

The first TBN formulations emerged decades ago and were directed towards its use on aircraft and missiles, but research on applying these methods to underwater vehicles is fairly recent. TBN for AUVs and submarines differs significantly from that of an aircraft with respect to the vehicle dynamics, but also to the sensors used. This section presents an overview of the necessary sensors for a correct perception

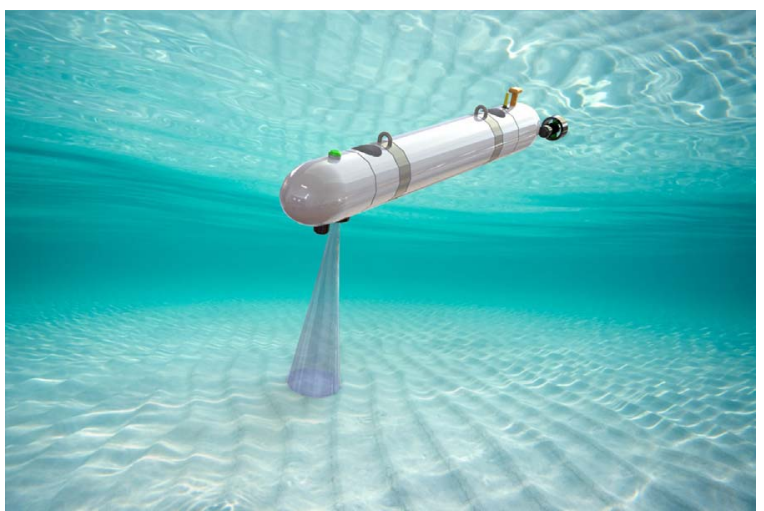

Fig. 2. Footprint of Altimeter sonar sensor.

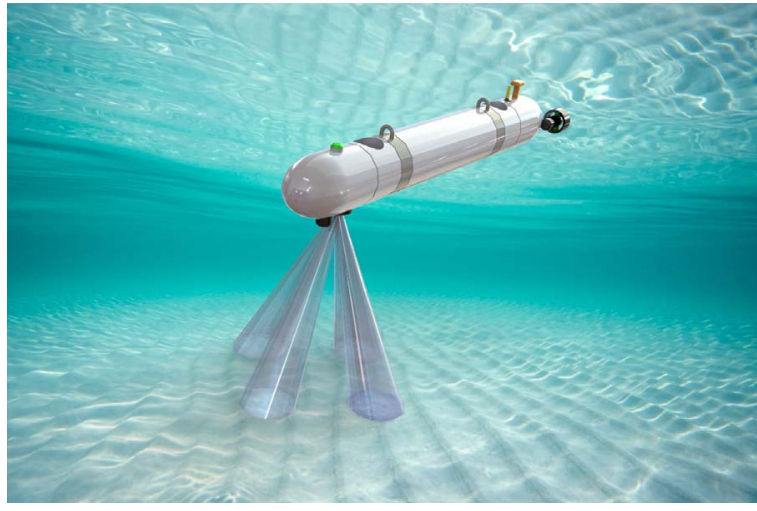

Fig. 3. Footprint of DVL sonar sensor.

of the terrain elevations. In fact, the achievable performance of any TBN system is determined primarily by sensor capability and also terrain variability. Thus, the sensors used, and their ability to correctly perceive the elevations and terrain variability is of uttermost importance Fig. 3.

TBN implementations for aerial vehicles usually require combining a barometric altimeter, outputting height above the mean sea level (MSL), and radar or laser altimeters, used to obtain the height of the vehicle from the terrain. A profile of the terrain in then obtained by combining the readings of these two sensors. Likewise, TBN implementations for underwater vehicles require the vehicle to be able to know its depth from the water surface, and its altitude towards the sea bottom, as described in one of the earliest works in the field by Massa and Stewart (1997). In this section a discussion on the different sensors that have been used for TBN solutions will be presented.

Underwater depth estimates are usually obtained by converting hydrostatic pressure, measured with a common barometric sensor, to depth using the UNESCO pressure-to-depth formula, presented by Fofonoff and Millard (1983). While hydrostatic pressure is affected by several environmental factors, like salinity, temperature or atmospheric pressure, these can usually be estimated in a fairly accurate way. Waves are also known to cause pressure fluctuations which negatively affect depth estimates, but if needed appropriate models can be used to filter out dynamic pressure sensor noise. The interested reader should refer to the works by Willumsen et al. (2007) and Hagen (2008) for more details.

Observing the sea-floor relief in underwater environments can be a greater challenge. Sonar sensors are the most reliable and commonly used sensor for underwater measurements of altitude towards the bottom. Sonar take advantage of the particularly good conditions of sound waves propagation in the water. Even though this behaviour is frequency dependent, sound waves are able to travel for long distances, in the order of kilometres, without significant attenuation. This is contrasting to the attenuation that light is subject to, which can only travel for a few meters before losing most of its energy, and limits the use of cameras or lasers to specific short-range applications. Therefore, for TBN the vehicles are required to be equipped with a sonar to image the bottom.

When a sound wave hits a portion of the ocean bottom, it is said to illuminate or ensonify that part of the bottom. A portion of the wave energy is absorbed by the sea floor, and the bulk of the energy that cannot be absorbed is reflected back into the water, in what is called an echo. Echo sounder sonars, also called active sonars, are devices that emit a pulse of sound, and then listen for the echo of the pulse reflected from the bottom, thus the designation. The time between the instant the sound is emitted until its echo is detected from the sonar, the timeof-flight (TOF) of the sound, is proportional to the range to the bottom Fig. 4.

Various kinds of active sonars have been used for TBN applications. 


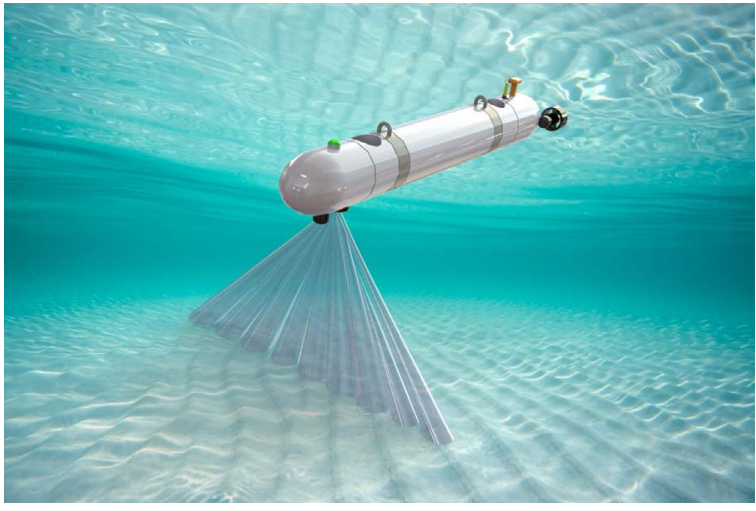

Fig. 4. Footprint of multibeam sonar sensor.

Expensive but highly-accurate sonar sensors, like Multibeam Echosounders (MBE), have been one of the main requirements for the success of TBN. However, recent work (Meduna et al., 2008, 2010; Meduna, 2011) has studied the performance trades associated with using lower quality cheaper sensors for TBN, for example Doppler Velocity Logger sonars (DVL) or single beam sonars. The use of a multiple sonar sensors combined for improved results has also been reported (Hagen et al., 2011). The differences between the different echo sounders are depicted in Figs. 2-4 and will be highlighted in the remainder of this section. Further details on sonar theory of operation can be found in both the Multibeam Sonar Theory of Operation (2000) and Manual on Hydrography (2011).

\subsection{Altimeter}

Similarly to laser or radar altimeters, commonly used on aircraft, sonar altimeters are instruments primarily used to measure the altitude, or height, above the sea bottom. Altimeters are single beam echo-sounders usually with narrow beam, of only a few degrees. Besides the transducer, altimeters employ basic signal processing to accommodate variations on the different properties of the environment, such as acoustic propagation and bottom reflection. They are also one of the cheapest option among the different kinds of sonars Fig. 5.

When compared to other sensors with multiple beams, altimeters have the drawback of requiring several consecutive measurements for disambiguation when applied to Terrain Based Navigation algorithms. Moreover, they are particularly sensitive to faulty readings, especially when the vehicle roll and pitch angles are high. Despite that, and because there are cheap sonars, there has been an increasing interest on using altimeter for TBN problems, for example the works by Williams and Mahon (2006); Meduna et al. (2008); Kim and Kim (2014).

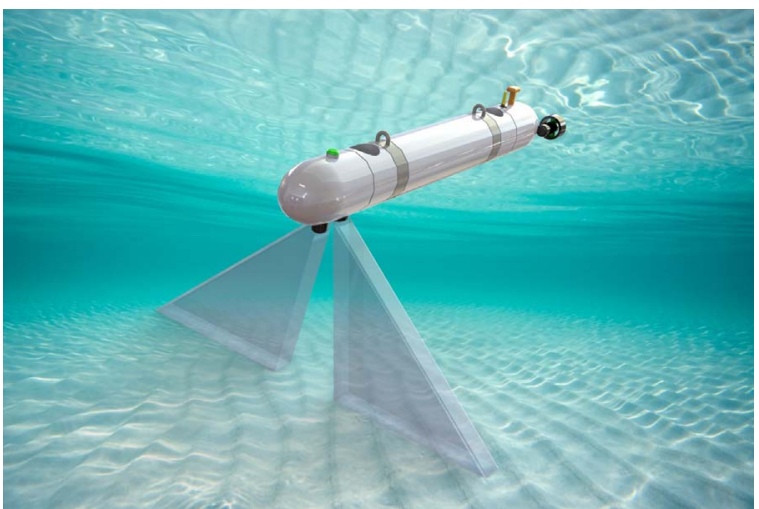

Fig. 5. Footprint of sidescan sonar sensor.

\section{2. $D V L$}

Doppler Velocity Loggers (DVLs) are one of the main sensors used by small-scale unmanned underwater vehicles, like AUVs and ROVs, to measure speed over ground. Usually mounted on the hull of the vehicles, DVLs make use of the Doppler effect that affects acoustic waves when reflecting on the bottom to calculate the velocity of the vehicle. The Doppler effect is the shift in frequency and wavelength of wave that is perceived by an observer moving relative to its source. The most common configuration for DVLs uses 4 beams pointing downwards, aligned with the fore, aft, starboard and port directions of the vehicle and each with an inclination of 20-30 degrees with the vertical, thus allowing for a three dimensional velocity vector to be derived.

Analogously to single beam sonars, DVLs can also provide altitude, or range to the bottom, by measuring the round-trip travel time of the pulses emitted by each of the beams. As DVLs have became quite popular as navigation sensors, the interest of using such sensors on TBN solutions arises naturally, and has been tried by different authors like Meduna et al. (2008); Donovan (2011), and others. Though not as cheap as altimeters, DVLs are still relatively cheap and are nowadays a fairly common navigation equipment in AUVs.

\subsection{Multibeam}

While Multibeam Echo Sounders (MBE) also rely on echo sounding to obtain terrain measurements, its working principle is, up to some point, distinct from the sensors covered on the previous subsections. MBEs have separated transducer arrays for transmission and reception, one projector and one hydrophone respectively. The first is oriented longitudinally and the latter is oriented transversally to the vessel's bow. By using advanced beam forming and beam steering techniques, it is possible for MEBs to ensonify multiple locations on the ocean floor with a single ping. The ensonified area, usually an area of points in a direction along track called a swath, is also covered with a higher resolution than conventional echo sounders. Naturally, MBEs are one of the most expensive and bulky kind of echo sonars. More details can be found in both Multibeam Sonar Theory of Operation (2000); Manual on Hydrography (2011).

A single MBE measurement can obtain the same terrain data points that multiple altimeter measurements performed at multiple locations. An MBE can have as much as 400 beams, or even more. According to Jalving et al. (2001) this feature makes MBE nearly optimal sonar sensors for bathymetric terrain navigation. Using such high number of beams is likely to introduce strong correlations between measurement errors of the different beams. To address such issue, Anonsen and Hallingstad (2006) proposed the adoption of sub-sampling procedure to effectively tackle this problem. Successful implementations of TBN algorithm using MBEs have been reported by numerous authors Anonsen and Hallingstad (2006); Anonsen et al. (2007); Anonsen and Hagen (2011); Donovan (2011). However, due to the high cost of these sonars, which can be many times that of a single-beam sonar, there is an increasing interest on using cheaper, and therefore less accurate, sensors for TBN solutions.

\subsection{Sidescan sonars}

The working principle of traditional sidescan sonars (SSS) is fundamentally different from the echo sounders described in the previous subsections. While the latter use the time-of-flight of acoustic waves to obtain terrain measurements, SSSs interpret not only the time-of-flight but also the acoustic backscatter intensity of the returned signal. After each pulse emission, SSS listen to the echo intensities at fixed time intervals, until a new pulse is emitted. The recorded data array, containing the received intensities is also referred to as swath. Naturally, the sampling period between two consecutive emissions determines the maximum sensor range. SSS are usually bulkier and 
Table 4

Main achievements for Underwater Terrain Navigation.

\begin{tabular}{|c|c|c|}
\hline Period & Achievements & Authors \\
\hline \multirow[t]{2}{*}{ 1990 2000 } & Initial developments of underwater TBN; & Bergem (1993), Massa (1997) \\
\hline & Real time implementations of the algorithms; & Massa and Stewart (1997) \\
\hline \multirow[t]{2}{*}{ 2000 2005 } & Measurement likelihood proven to be Gaussian; & Karlsson et al. (2003), Nygren and Jansson (2004) \\
\hline & Initial studies on the CRLB of TBN estimators; & Nygren (2005) \\
\hline \multirow[t]{2}{*}{ 2005 2010 } & Nonlinear Bayes Filter for underwater TBN; & Anonsen and Hallingstad (2006), Kjetil (2007) \\
\hline & Initial studies of the CRLB of TBN estimators; & Meduna et al. (2008), Donovan (2011) \\
\hline \multirow[t]{2}{*}{ 2010 2015 } & Tighly-coupled integration between TBN and INS; & Williams and Mahon (2006), Anonsen (2010) \\
\hline & Use of low-accuracy bathymetric sensors (DVL, altimeter) & Meduna (2011), Donovan (2011); \\
\hline
\end{tabular}

require additional availability of energy and processing power, which may be not compatible for use with some classes of AUVs.

Typical SSS are characterized by having two sensing heads, aligned with port and starboard directions, usually with an angle of around 45 degrees to the vertical. Hence, SSS have a wide swath even at low altitudes. This can be particularly advantageous in shallow water scenarios, where the footprint of other sensors would necessarily be small. The emitted beams are usually narrow, and across the direction of movement. Therefore, two-dimensional acoustic images of the seabed can be obtained by acquiring successive swaths of data, but this necessarily requires that the sonar is in motion. Consequently, the motion and attitude that the sonar undergoes needs to be taken into account when processing the obtained data.

The acoustic echo intensity is mainly influenced by the reflectivity of the sea floor, but it is also attenuated depending on the travelled distance and corrupted by the SSS ensonification pattern (Burguera and Oliver, 2016). Therefore, instead of producing bathymetric data, SSS provide a relatively high definition acoustic image of the seabed, which can be interpreted as a monochromatic representation of the physical properties of the bottom. However, the data obtained by SSS is not an accurate visual representation of the sea floor, as the intensity returns are functions of multiple factors like the grazing angle, terrain surface, and local water properties. For example Wilby (1999) reported that one of the drawbacks of using SSS is that gentle undulations in the seabed are usually perceived as a flat area. Moreover, it is customary to have parts of the bottom occluded due to terrain elevations. Therefore, applying standard image-processing techniques to the produced images is anything but straightforward. Because of that, SSS have been used in solutions of AUV localization and navigation, but only in the context of landmark detection and matching, particularly in the case of existing known local features.

Because SSS do not provide bathymetric information of the terrain, their use for TBN has been rather scarce. However, recent studies have investigating the feasibility of using SSS as a primary sensor for TBN (Padial et al., 2014a, 2014b). The presented method tries to directly correlate SSS acoustic shadows with bathymetric maps, by formulating an appropriate measurement model that takes as inputs a map and vehicle pose, and outputs an expected sidescan intensity returns. The predicted SSS shadows are then correlated with the actual SSS data in order to obtain a possible positioning aid.

Leveraging from advanced signal processing techniques, different kinds of SSS have emerged that are likely to play a more important role on bathymetric tasks. Examples of this are the Synthetic Aperture Sonars (SAS), also known as Phase Differencing Bathymetric Sonar. The principle behind SAS is to combine successive pings coherently along a known track in order to increase the along-track resolution (Hansen, 2011). In that way, SAS can potentially produce high resolution images, which is great for applications like searching for small objects or imaging the seafloor. If additionally SAS are equipped with two receiver arrays, vertically displaced, it is then possible for an interferometric processing of the returned echos. In that way, it is possible for the SAS to provide not only the usual high-resolution data from traditional SSS, but also able to retrieve bathymetric information.
The interested reader should refer to the work by Hansen et al. (2003) for an overview of signal processing chain required for AUV based sonar interferometry. Naturally, interferometric SAS can be used as primary bathymetric sensors for TBN, as reported by Stalder et al. (2008); Hagen et al. (2011); Hansen et al. (2011).

\section{Underwater TBN}

TBN for underwater environments is fairly recent, at least when compared to aerial techniques. The main differences between the methods developed for the two environments are mostly related to the sensors used and, naturally, the vehicle dynamics. While aerial TBN has been focused mostly on the use single beam sensors, usually radar or laser altimeters, the underwater community has been focused on using dense sensors, able to map large areas of terrain within a single measurement acquisition step. In this section, a brief overview of Terrain Based Navigation for underwater vehicles will be provided, focused on the most prominent approaches. Table 4 summarizes the main achievements of TBN for AUVs since the early days.

Studies detailing a possible TBN system for AUVs were initially described by ,. Even though it was just a description of a possible system, the author had already anticipated underwater terrain navigation to be a much more difficult problem, namely in terms of collecting the necessary data for terrain navigation and, furthermore, that data may have large errors. As described, the existence of different layers on the water, like thermoclines, pycnoclines and haloclines, that result from the natural properties of the water, could seriously affect the data collected.

Later on, Bergem (1993) studied the problem of bathymetric navigation of AUVs using a multibeam sonar. It was the first thorough evaluation of TBN for AUVs, and the demonstration that navigational errors could be bounded by only using information from bottom topography. Despite being only an initial work in the field, the work presented by Bergem also gave some insight on some important theoretical aspects. Example of this is the development a method to compute a Relative Measurement Covariance Matrix, adequate to the use of multiple beam sonar measurements. His approach is built around a Kalman Filter and was implemented and tested with real data. Massa (1997); Massa and Stewart (1997) focused on some aspects of TBN that had been left undealt by previous approaches. First, the possibility to deal with multiple matches in the correlation algorithm was addressed. This issue was achieved by applying probabilistic data association techniques. Additionally, some effort was directed towards the real-time implementation of the algorithms. Specifically, a coarse-to-fine algorithm was introduced to intelligently search for good matches. Also, in order to alleviate the computational requirements, suitable validation gates for the correlation outputs were also derived.

Following to that, a series of papers by Lucido et al., (1998, 2004) dealt with the problem of matching high resolution local depth maps against a large, on-board, low resolution reference map. The focus was on the use of invariant of special critical points and also on the multiscale analysis of bathymetric maps. Probably due to development 
and improvement of sonars, the work presented was not followed by any of the subsequent articles in the field of TBN.

Early classifications divided TBN methods into two different classes, namely the batch methods and the sequential methods, as previously detailed in Section 3. Regardless of that, Anonsen (2010) introduced an alternative classification of underwater terrain navigation methods, dividing them between Search Area methods or Gradient-Based methods. This new designations are related with how terrain measurements are incorporated on the navigation filter of the vehicle. Indirectly, they are also a consequence of the ability to process large swaths of terrain in a single step, contrary to the traditional batch methods. This is done using complex Bayesian Estimation techniques, as will be covered ahead on Section 7 .

Loosely speaking, TERCOM tries to correlate the entire swath of measurements of the terrain with profiles of the map, using the best result of such match as a system measurement. Hence, in TERCOM a search is made on the entire map, or part of it, to find the position that best matches the entire terrain measurements swath. On the other hand, in SITAN like methods the multiple depth measurements provided by the sonar are used to compute local gradients and propagate the estimated position in the direction indicated by the measurements. Having this in mind, TERCOM could be classified as a Search Area method while SITAN would be a Gradient-Based methods.

On an alternate perspective, TBN algorithms can also be classified according to the degree of integration with the INS: we can distinguish between loosely and tightly coupled integrations. According to Hagen et al. (2011), in the tightly coupled approach the bathymetric measurements are used directly within the filter of the INS, along with all the other sensor measurements. On the other hand, in the loosely coupled approach, the bathymetric measurements are processed in a parallel filter until it converges or diverges, and in case of convergence, the position estimate from terrain navigation is fed back to the INS as a regular position measurement. Nygren and Jansson (2004); Nygren, (2005, 2008) developed some work of paramount relevance, on which the use of a 3D sonar for the subject of correlation-based terrain navigation was analysed. One of the most significant results was the theoretical proof that, under certain conditions, the Maximum Likelihood estimator is optimal, and that the likelihood measurement function asymptotically converges to a Gaussian probability distribution as the number of measuring beams increases. The Cramér-Rao lower bound, which is commonly used to expresses a lower bound on the variance of estimators, was also thoroughly discussed for underwater TBN problem. Additionally, the actual implementation and test of the approach on an AUV using a multiple beam area-sonar were also presented. Even though the position error was a bit far from the $1 \mathrm{~m}$ RMS predicted by the simulations, the corresponding error in the seatrial was of only a few meters, which is very impressive considering the ground truth given by DGPS had accuracy of about $3 \mathrm{~m}$ RMS (see Nygren, 2005).

TBN is inherently a strong non-linear problem, mostly because of the non-linear nature of the terrain measurement function. As such, the interest on using non-parametric nonlinear Bayesian methods to address the problem of underwater terrain navigation is obvious. Following the initial theoretical work by Bergman (1999), several authors from the Norwegian University of Science and Technology, most notably Anonsen and Hallingstad (2006); Anonsen and Hagen (2011) among others, presented a number of different estimation algorithms applied to the TBN problem. Details on the different Bayesian Estimation techniques, like Particle Filters, Point-Mass Filters and Sigma Point Kalman Filters were studied, and their performance was compared using real AUV data. According to the authors, the Point-Mass Filter is in general slightly more accurate and robust than the Particle Filter, but also more computationally expensive. Details on the different Bayesian Estimation filters will follow in Section 7. Moreover, with a series of sea trials, the authors demonstrated the ability to achieve real-time TBN using a MBE, having obtained bounded errors within 5-20 m, with the vehicle navigating for a period of more than two hours.

Since the early days, the use of TBN for underwater vehicles has been highly focused towards the use of powerful multiple beam sonar, able to provide a high resolution perception of the environment. Experimental validation of such approaches was also consistently coupled with the use of high-grade INS. Lately, some research efforts have been directed on a rather opposite approach, which is the application of TBN for sensor limited systems. Such denomination, introduced by Meduna (2011), refers to a class of vehicles equipped with a combination of low-accuracy inertial sensors and simple, lowinformation sonar like DVLs or altimeters. The use of DVLs or single beam sonars for TBN purposes has also been reported by other authors Morice et al. (2009); Donovan (2011). Since the larger group of existent AUVs is, by far, equipped with such low accuracy sensors, the rational of such approach is evident.

Meduna et al., (2008, 2010); Meduna (2011) developed a tightlycoupled TBN filtering framework for sensor-limited vehicles, which basically relies on the estimation of critical sensor errors, tightly integrated with the on-board navigation system. The success of the approach was also experimentally demonstrated in different scenarios like long range navigation and return-to-site missions. An analytical formulation was developed giving a conservative prediction of the terrain navigation estimate sensitivity to constant attitude errors. A comparison between the performance achieved using different range sensors like MBE, DVL and altimeter, has also been provided. During a series of field trials in Soquel Canyon, Monterey Bay, terrain correlations were performed using the Benthic Imaging AUV (BIAUV) with DVL sonar range measurements and a $1 \mathrm{~m}$ resolution bathymetry map generated by MBARI's Mapping AUV (MAUV). The authors reported that accuracy and precision of 5-10 $\mathrm{m}$ was achieved, which was shown to be only a factor of two worse than the performance achieved when using the significantly more expensive, sensor-rich MAUV.

Claus and Bachmayer (2015) presented results documenting the use of a TBN method for an underwater glider. The algorithm used, based on a Particle Filter, makes use of the vehicle's dead-reckoned navigation solution, altimeter, and a local digital map, and it was experimentally validated with both offline and online field trials. The offline trials allowed to adjust a number of parameters like the jittering variance, the DTM grid cell size, or the number of particles. In both of them the TBN algorithm provided bounded error location estimates that improved on the dead-reckoned estimates.

One of the main factors contributing to the success of TBN systems is the amount of terrain variability. As initially postulated by Massa and Stewart (1997), results demonstrate a more accurate navigation in areas with greater bathymetric variability occurs, opposed to flatter areas with more gentle terrain contours. Anonsen (2010) claimed that TBN occasionally converges to overconfident, incorrect solutions when operating for an extended period of time over featureless terrain. Noting that, Dektor and Rock (2012) analysed the causes for the filter failure when in flat terrain. According to the author, the cause of false fixes in information-poor regions is the assumption that the terrain is uncorrelated. Dektor and Rock (2014) further addresses this issue by making some adaptive variance adjustments, which is dependent on the amount of map error, sensor error, and information of the terrain. Houts et al. (2012) designed a robust framework for failure detection and recovery for TBN. The framework builds up on a series of diagnostic checks and, in particular, by checking whether the measurements predicted by the estimate agree with the observed measurements.

On a complementary line of work, Hausler et al. (2013) developed path planning algorithms that along choose a suitable vehicle path that maximizes the amount terrain information. His approach involves on an initial phase an instantiation of an $A^{*}$ algorithm, on which the cost criterion to maximize is related to the integral of the magnitude of the terrain gradient along its trajectory. After that, and using an optimal 
control formulation, the initial trajectory is refined taking into account several constraints, like the vehicle dynamics or collision avoidance schemes. Furthermore, TBN performance is not only dependent on the actual terrain variation, but on numerous factors, like algorithm characteristics, sensor accuracy, map accuracy and map resolution. A discussion of the map resolution effects on the performance of TBN algorithms can be found in Meduna (2011). Kedong (2010) discussed the influence of different conditions like terrain characteristics, map resolution, initial INS error and others on the precision of underwater TBN.

\section{State space model}

A generic continuous-time stochastic filtering problem can by described in a dynamic state-space form according to the following equations (Chen, 2003):

$\dot{x}_{t}=f\left(t, x_{t}, u_{t}, w_{t}\right)$

$y_{t}=g\left(t, x_{t}, u_{t}, v_{t}\right)$

In (2) $x_{t}$ refers to the state variable vector, $u_{t}$ refers to the system inputs, and $t$ denotes time. $w_{t}$ and $v_{t}$ represent process noise and measurement noise, respectively. Despite its simplicity, the above model is rather general and can be applied in most of the applications. The function $f\left(t, x_{t}, u_{t}, w_{t}\right)$ describes the system dynamics, and determines how the states evolve over time. On the other hand $g\left(t, x_{t}, u_{t}, v_{t}\right)$ is the measurement equation and describes how observations of the system are made, and how do they relate to the system state, $x_{t}$. The process and measurement noise components are generally unknown and modelled as stochastic processes.

The state-space approach is a natural framework to address TBN. In what follows we will present the different formulations for the TBN systems that can be found throughout the literature for both the process and measurement models, in an unifying perspective. There will be a particular emphasis on the different flavours for both the process and measurement models employed on the most relevant approaches from the literature.

From (2), a basic discrete state-space model for an INS-based AUV system, can be expressed by the following difference equations:

$x_{k+1}=f\left(x_{k}, u_{k}\right)+w_{k}$

$z_{k}=g\left(x_{k}\right)+v_{k}$

The difference Eqs. (3) can capture the fundamental idea of the general TBN approaches, on which vehicle terrain elevation data is used to correct the existing drift of INS. The characteristics of such problem are described by both the state transition equation (3a) and measurement model equation (3b), that will be further described the following subsections.

In most of the early approaches, only motion on the North-East plane was assumed. This means that no uncertainty in any other directions can be observed and, therefore, there is no need to estimate them. While such assumptions are rather strict and difficult to observe, especially when in presence of sensor limited systems, they were introduced, up to a great extent, due to the computational requirements.

$x_{k}=\left[\begin{array}{ll}x_{N, k} & x_{E, k}\end{array}\right]^{T}$

$u_{k}=\left[\begin{array}{ll}\Delta x_{N, k} & \Delta x_{E, k}\end{array}\right]^{T}$

A problem with the state space in (4) is that uncertain depth measurements might arise due to tidal levels variations. This can negatively affect the results obtained by TBN algorithms, particularly on missions with several hours of duration. To counter this, the time varying tidal level needs to be estimated. Anonsen and Hallingstad (2006) added a depth bias term, related to tidal errors, to the state vector, in order to address the non-negligible effects of tides when determining the altitude of an AUV with respect to the sea bottom. A similar approach is also suggested by Donovan (2011). Alternatively, it was also possible to work with relative profiles, but this option must be carefully considered as it is known to perform poorly in self-similar terrains.

With the increase of available computational power on-board the vehicles, different authors have proposed to increase the state space model to cope with different characteristics of the TBN problem. This has the purpose of trying to incorporate prior knowledge and taking advantage of specific robot configurations. Another reason for the use an augmented state vector is the use of sensors with poor accuracy, thus requiring the respective state variables to be estimated, as concluded by Anonsen et al. (2007). Meduna et al. (2010) introduced significant changes to traditional underwater TBN systems: the state vector is augmented to a dimension of eight, and being composed not only by the vehicle position, but also by the vehicle attitude and angular rates. This also motivates and tightly-coupled integration of the acquired bathymetric measurements.

\subsection{Motion model}

Eq. (3a) represents the state transition equation. The state vector, $x_{k}$, is assumed to be Markovian, usually representing the vehicle's 2dimensional horizontal position, at time instant $k$. These positions are frequently referenced to a north-east-down earth-fixed frame. $u_{k}$ contains the position updates as calculated from the INS, and $v_{k}$ represents the noise associated to the updates.

In the literature, there has been a preference for having the state space model, described by (3a), to follow simple linear models. This is what happens for example in Anonsen et al. (2007); Donovan (2012), to mention only a few. Nevertheless, depending on the available onboard sensors and on-board computational power, other models can also be used, particularly in the cases when a tight-integration with the INS is performed.

According to Donovan (2011), the stochastic motion model errors are dominated by the INS drift error, and commonly characterized as an independent white Gaussian sequence. Some references also mentioned the use of the Singer model, a zero-mean stationary first-order Markov process. This is justified when it is necessary to capture the trongly correlated propagation error of the INS (see Bergem, 1993; Anonsen et al., 2007).

\subsection{Measurement Model}

Recalling (3b) from above, $g\left(x_{k}\right)$ is a non-linear function that retrieves from a map the total depth, or altitude towards the bottom, for the current vehicle position. This is fact just the vertical distance from the surface to the bottom. For the general case, the vehicle is not navigating at the surface when acquiring the range measurements, therefore the actual depth of the vehicle, $d_{k}$, must also be taken into account. The depth $d_{k}$ can be measured with relatively high precision by common pressure sensors. This situation is illustrated in Fig. 6, where the case of an AUV carrying a single-beam altimeter is depicted, and can be described as:

$z_{k}=\mathbf{M}\left(x_{k}\right)-d_{k}+v_{k}$

In the equation above, $\mathbf{M}\left(x_{k}\right)$ refers to the function that provides the depth for a given position, obtained from a DTM. On its most common form, DTMs consist on gridded nodes where each node corresponds to a depth at a specific location. The grid is usually equally spaced, and depth at locations other that the grid nodes are obtained through bilinear interpolation. Nygren (2005) claimed that in relatively flat areas using linear interpolation is appropriate, but in more rough hilly terrains a more accurate method must be used. However, in a more recent work, Meduna (2011) stated that higher-order interpolation methods give small performance gains at the expense of large 


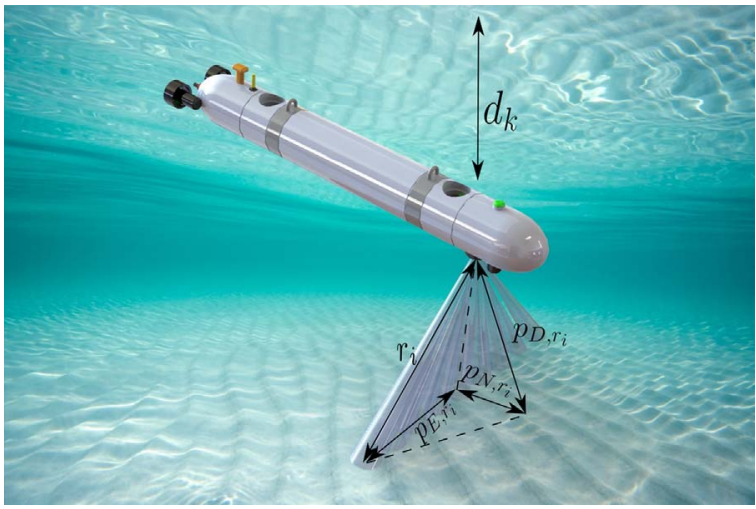

Fig. 6. Schematic view for the terrain measurements for the single beam sonar case

computational effort and that such higher order methods are more susceptible to overfitting. Such situations can lead to an over-estimation of terrain variability and consequently degrade the performance of the system. Anonsen (2010) provided insight on problems that can arise due to map representation issues.

The vector of observations consists on range measurements, as obtained by the bathymetric sensor employed. For single beam sensors, the observation vector consists of only a single range measurement, but for multiple beam sensors the observation vector will consist on the different $r_{i}$ range measurements stacked in a vector, as in (6).

$\mathbf{z}_{k}=\left[\begin{array}{lll}r_{1}\left(x_{k}\right) & \ldots & r_{N}\left(x_{k}\right)\end{array}\right]^{T}$

The likelihood of a given range measurement $r_{i}$ can be obtained by comparing it with the bathymetric map of the area $\mathbf{M}\left(x_{k}\right)$, naturally affected by $d_{k}$. When the vehicle has non-negligible roll and pitch angles, the range measurement obtained needs to be properly compensated for it. The same happens when sonar sensors have multiple beams with a large swath. This is of particular importance when the vehicle is navigating at high altitudes. Most TBN implementations use projection-based methods for that. Each measured range, $r_{i}$, is projected into the three-dimensional space:

$\left[\begin{array}{l}p_{N, r_{i}} \\ p_{E, r_{i}} \\ p_{D, r_{i}}\end{array}\right]=R(q) a\left(r_{i}\right) r_{i}$

In (7) $R(q)$ refers to the rotation matrix for the specific vehicle orientation $q$, and $a\left(r_{i}\right)$ refers to the unit direction vector of beam corresponding to $r_{i}$. By doing so it is possible to compute the location $p_{r_{i}}$ where a specific beam hit the bottom of the sea, and from there evaluate the likelihood, as it will be made clear ahead. While the notation here used is adapted from Meduna (2011), similar approaches can be found elsewhere. According to the same author range-based schemes, on which range measurements are compared directly with a predicted range, can also be employed However, as such alternatives are based on ray-tracing techniques, which can be computationally demanding, they tend to incompatible with the processing power available on-board the vehicles.

Analogously to the motion model, stochastic measurement model errors are described by an independent white Gaussian sequence, $v_{k} \sim \mathcal{N}\left(0, \sigma_{k}\right)$. However, and as detailed by Hagen et al. (2011), the fusion of different sensors can motivate a more detailed error model, that can capture the characteristics of each individual sensor, namely AUV depth errors $v_{z}$, attitude and alignment errors $v_{a}$, sound speed errors $v_{c}$, DTM errors $v_{h}$ or even sensor processing errors $v_{s}$.

Combining (5-7), the final measurement model (8) is obtained:

$p_{D, r_{i}}=\mathbf{M}\left(\left[\begin{array}{l}x_{N, k} \\ x_{E, k}\end{array}\right]+\left[\begin{array}{l}p_{N, r_{i}} \\ p_{E, r_{i}}\end{array}\right]\right)-d_{k}+v_{k}$

Assuming that the measurement errors in each of the beams to be uncorrelated and independent, the likelihood of a measurement $\mathbf{z}_{k}$, composed out of $N$ range measurements, is given by

$p\left(\mathbf{z}_{k} \mid \mathbf{x}_{k}\right)=\alpha \exp \left(-\frac{1}{2} \sum_{i=1}^{N} \frac{1}{\sigma_{k_{i}}}\left(r_{i}-p_{D, r_{i}}\right)^{2}\right)$

where $\sigma_{k_{i}}$ refers to the covariance of the measurement error for beam $i$, assumed independent and identically distributed Gaussian, while $\alpha$ is just a normalization constant.

\section{Bayesian estimation}

Bayesian filters have become the standard for sensor fusion in integrated navigation systems. Morice et al. (2009) stated that the strength of Bayesian filtering techniques is that they allow the fusion of information from multiple sensors, taking into account both the sensor measurements and the accuracy of the sensors. Moreover, these probabilistic algorithms also allow to explicitly deal with uncertainties in both the motion and the measurement models. This section will briefly review the different Bayesian Estimation algorithms that have been used for Terrain Based Navigation for underwater vehicles. For details on the theoretical derivation of such filters, please refer to works by Bergman (1999); Arulampalam et al. (2002); Chen (2003), and the references therein.

The objective of an estimation procedure is in general to obtain the value of a parameter $x$, given an observation of an experimental outcome $y$. This has been mathematically stated most notably as the Bayes theorem:

$p(x \mid y)=\frac{p(y \mid x) p(x)}{p(y)}$

Bayesian Estimation adapts the Bayes theorem to a statistical paradigm. In the Bayesian Estimation framework all the unknowns are considered a stochastic variable, described by its respective probability distribution. The goal of Bayesian Estimation is then to compute the posterior distribution of the state vector $\mathbf{x}_{t}$ of a stochastic system, given a set of observations $\mathbf{z}_{t}$.

At the core of Bayesian Estimation framework is the Bayes Filter, a general probabilistic tool that has been extensively. Under Markovian and mutually independence assumptions, and using the Bayes formula (10) and the law of total probability, the general Bayes Filter can be approximated by the following Bayes Filter recursion:

$p\left(\mathbf{x}_{t} \mid \mathbf{z}_{t-1}\right)=\int p\left(\mathbf{x}_{t} \mid \mathbf{x}_{t-1}\right) p\left(\mathbf{x}_{t-1} \mid \mathbf{z}_{t-1}\right) d_{\mathbf{x}_{t-1}}$

$p\left(\mathbf{x}_{t} \mid \mathbf{z}_{t}\right)=\frac{p\left(\mathbf{z}_{t} \mid \mathbf{x}_{t}\right) p\left(\mathbf{x}_{t} \mid \mathbf{z}_{t-1}\right)}{p\left(\mathbf{z}_{t} \mid \mathbf{z}_{t-1}\right)}$

$p\left(\mathbf{z}_{t} \mid \mathbf{z}_{t-1}\right)=\int p\left(\mathbf{z}_{t} \mid \mathbf{x}_{t}\right) p\left(\mathbf{x}_{t} \mid \mathbf{z}_{t-1}\right) d_{\mathbf{x}_{t}}$

Eq. (11) corresponds to the time update equation, and this is determined by the state transition density, $p\left(\mathbf{x}_{t} \mid \mathbf{x}_{t-1}\right)$. On the other hand, Eq. (12) is the measurement update equation, and is determined by the measurement model, $p\left(\mathbf{z}_{t} \mid \mathbf{x}_{t}\right)$ and the prior, $p\left(\mathbf{x}_{t} \mid \mathbf{z}_{t-1}\right)$. The term $p\left(\mathbf{z}_{t} \mid \mathbf{z}_{t-1}\right)$ is a normalization constant, evaluated as in (13).

When the dynamical system has a state space representation as in (3), $p\left(\mathbf{x}_{t} \mid \mathbf{x}_{t-1}\right)$ and $p\left(\mathbf{z}_{t} \mid \mathbf{x}_{t}\right)$ can be easily computed if the noise densities $w_{t}$ and $v_{t}$ are known, as follows:

$p\left(\mathbf{x}_{t} \mid \mathbf{x}_{t-1}\right)=p_{w_{t}}\left(\mathbf{x}_{t}-f_{t}\left(\mathbf{x}_{t-1}, u_{t}, t\right)\right)$

$p\left(\mathbf{z}_{t} \mid \mathbf{x}_{t}\right)=p_{v_{t}}\left(\mathbf{z}_{t}-g\left(\mathbf{x}_{t}, t\right)\right)$

The Bayes Filter is not analytically solvable for the general case, mostly due to the complexity involved on evaluating the existing integrals. TBN can be implemented using one of the different realizations of the Bayesian Filter namely parametric filters like the Kalman Filter (KF), the Information Filter (IF) or even the Extended Kalman 
Filter (EKF), or non-parametric approaches like the Particle Filter (PF) or the Point Mass Filter (PMF).

The earlier approaches for underwater TBN were characterized by the use of a KF to address the navigation problem. The Bayes Filter can be optimally implemented as a KF under the assumptions that both the state transition model and observation model can be represented by means of a linear stochastic equation, and additionally that the state and process noise are mutually independent Gaussian distributions. While these assumptions can be considered strong, especially considering that TBN is inherently non-linear, several authors proposed the use of a $\mathrm{KF}$ to address the problem of underwater terrain navigation, most notably Bergem (1993) and Massa (1997). In such approaches the measurement inputs of the KF are the position outputs of a given correlation function, as it typical on Search-Area methods. Such approaches have low computational requirements, when compared to its non-linear counterparts..

Gradient-based methods, on the other hand, have its roots on the EKF. EKFs are able to deal with non-linear problems by using an approximation of both state transition and observation model. These approximations are based on the first-order Taylor series expansion around a given working point. This is particularly convenient when dealing with Gradient-based methods which use terrain depth measurements directly as an input to the filter, as described on early SITAN formulations by Hostetler (1978) and Hollowell (1990). However, the Taylor expansion requires the computation of the terrain gradient vector which is sometimes non-trivial, particularly when in the presence of highly non-linear environments. In such cases, the computed gradient can negatively influence the accuracy of the approximation, and therefore result in poor TBN estimates. EKFs have also been used by Yuan et al. (2012) in methods that combine both gradient based and search-area methods.

The non-linearity of the process described by (3) motivates the use of a full Bayesian, non-parametric filter for successfully tracking the vehicle state estimate. However, while those are in general more accurate in representing non-linear systems, and relatively simple to implement, its main drawback is related with their computational complexity, that quickly increases with the dimension of the state space.

The Sigma Point Kalman filter (SPKF), also known as Unscented Kalman filter (UKF), is a nonlinear realization of the Bayes filter that relies on the use of only a few deterministically chosen sigma points, which are then propagated through the nonlinearities of the process and measurement equations to yield a new sigma point approximation of the posterior density. A navigation filter based on the unscented transform was briefly sketched by Nygren (2005), who concluded that the SPKF to be a non-optimal filtering method that fits the problem of TBN. Later, the performance of SPKF using real AUV data was also analysed by Kjetil (2007). However, due to is characteristics and simplicity, non-parametric realizations of the Bayes Filters, like the Particle Filter or the Point Mass Filter have gained more attention in the recent approaches.

The Particle Filter, also sometimes referred to as Sequential Monte Carlo (SMC) methods, is a numerical approximation to the Bayesian filter for non-linear systems. The PF method uses a large number of hypothetical samples of the state vector to estimate its probability distribution. Each sample is called a particle, hence the name, and they are generated according to its probability density function (PDF). Each of the particles is then weighted according to the most recent observation. The key idea in PF is to represent the required posterior density function by a set of random samples an its associated weights. It can be demonstrated that as the number of samples becomes very large, this discrete weighted approximation becomes an equivalent representation to the usual posterior density. The particle filter is also known to be asymptotically optimal in a minimum mean square sense. Various authors have reported the use of PF for underwater terrain navigation. Examples of this are the works by Bachmann and Williams
(2003); Karlsson et al. (2003); Williams and Mahon (2006); Nakatani et al. (2009); Morice et al. (2009); Meduna et al. (2010); Murangira et al. (2011); Stuckey (2012); Melo and Matos (2013).

The Point Mass Filter is yet another non-parametric realization of the Bayes Filter, even though not as popular as the PF. In this case, the posterior density is assumed to be represented by a set of point masses, ordered in a grid. The continuous PDF is obtained by integrating over the masses of the grid. Among the main advantages of such filters is their ability to solve the Bayesian Filter in an asymptotically optimal way. Even though the size of the grid is usually fixed, efficient implementations of the PMF use grid adaptation mechanisms to automatically adjust the grid mesh, which is particularly useful in situations when a high gradient PDF exist. This grid-adaptation mechanism can also be regarded as a trade-off between algorithm performance and computational requirements. Examples of the use of PMFs for underwater TBN were given by Jalving et al. (2001); Carlström (2007); Jian et al. (2012). Hagen et al. (2011) proposed the used of a PMF to address the problem of TBN for AUVs navigating at low altitude.

Both PFs and PMFs have been successfully implemented for underwater TBN and studies comparing these two implementations have also been performed. Anonsen and Hallingstad (2006) and Meduna et al. (2008) showed that the PMF is more robust and accurate than the PF, whereas the PF allows higher dimensional search without the computational expense of the PMF.

Non-linear filters like the Particle Filter or the Point-Mass filter, previously described, can gracefully address the non-linear problem of TBN. However, these filters tend to scale very poorly when the number of dimensions of the state space increases, which tends to happen more frequently when dealing with sensor-limited systems. In such situations, the Rao-Blackwellized Kalman Filter (RBPF), also known as Marginalized Particle Filter is of great use. The RBPF is a powerful combination of a PF and a KF, which can be used when the underlying model contains a linear sub-structure, subject to Gaussian noise. In such filters, the states following a linear model can be estimated with the $\mathrm{KF}$, while the non-linear states are estimated with the PF. The use of RBPF for TBN has been suggested by Schon et al. (2006) and Nordlund and Gustafsson (2009), however these studies were mostly focused on the estimation point of view of the problem, and did not consider the peculiarities of underwater TBN. Anonsen (2010) suggested that a RBPF could be used to gain a better understanding of the overconfidence effects, possibly by integrating the TBN updates more tightly in the inertial navigation system. Teixeira et al. (2012) made a decisive contribution by formally deriving two variants of a RBPF and assessing their performances when applied to the underwater TBN problem in particular.

When dealing with estimation, it is always convenient to establish a lower bound on the estimation error that is possible to achieve. According to Bergman (1999), such a bound can then be used to quantify the fundamental performance level that can be reached for the currently studied estimation problem. The Cramér-Rao Lower Bound (CRLB) is known to set a lower limit on the mean square estimation error of every estimator. In the context of TBN, the CRLB is often used to assess the performance of the filter, by evaluating how far from the CRLB the obtained estimates are. In this way, the CRLB is also an useful tool when designing INS, establish sensor performance requirements, or even estimating the amount of terrain information needed to achieve a given performance level. An initial theoretical insight on the CRLB applied to the underwater TBN was provided by Bergman (1997). The analysis of the CRLB for different navigation solutions for TBN was also covered by Karlsson et al. (2003) and Nygren (2005).

\section{Related work}

So far this article dealt exclusively with the problem of TBN, which has been detailed in the previous sections. TBN has been described as 
the process of localizing a robot in the environment with the aid of known a-priori map of the terrain. However, what to do when these terrain maps are not available, as it happens in the majority of the situations? Considering this, several authors have adopted and extended Simultaneous Localization and Mapping (SLAM) techniques to underwater vehicles.

\subsection{Underwater $S L A M$}

Even though previous authors have provided early descriptions of the SLAM problem, Csorba (1997) was the first to present a rigorous theoretical investigation into the SLAM problem. According to him, the SLAM problem could be presented as the ability of having an autonomous vehicle, deployed at an unknown environment, and then have it build a map and use this map to navigate. Being a fundamental problem for the mobile robotics community, early SLAM algorithms have since then been extended into a variety of application-dependent alternatives.

The traditional approach for the SLAM problem uses an EKF for estimating both the vehicle's pose and the position of the different features. This means that the state vector needs to be augmented accordingly, to accommodate all the necessary information. As the number of features to track increases, the estimation process becomes complex and demanding to solve in real time. Particle Filters could also be a very appealing option, as both process and sensor noise can be modelled by non-parametric probability density functions. However, as stated by Thrun et al. (2005), a straightforward implementation of this filter for the SLAM problem would be doomed to fail, due to the large number of variables involved in describing a map. Nevertheless, with some adaptations, PF based SLAM algorithms have also been proposed, namely the FastSLAM algorithm by Montemerlo et al. (2002), the Distributed Particle SLAM (DP-SLAM) by Eliazar and Parr, (2003, 2004), or the Segmented SLAM by Fairfield et al. (2010).

SLAM has been widely used in ground field robotics, but ts use in underwater environments is not so widespread. The sub-sea domain is arguably one of the most difficult environments for the use of SLAM techniques, mostly due to the lack of natural features. This is particular evident for underwater unstructured environments. In addition, the sub-sea sensors, such as sonar or cameras, used to extract these features or navigation landmarks provide very weak or ambiguous sensory information. The detection of underwater features is a field which has obviously been given some attention by different authors. For example Aulinas et al. (2011) addressed this issue by using optical camera images, and Aulinas et al. (2010); Woock (2012) focused on the use of sonar images. Makumder (2001) developed the concept of blob, used to refer to an entity which is repeatedly, consistently and invariantly detected by a sensor in a spatial region. This is in a way a generalization of the concept of a feature which can accommodate a large number of characteristics. It also facilitates the modelling of unstructured sub-sea landmarks, and their inclusion in a generalized probabilistic model.

The real-time requirements for actual implementation in AUVs are in fact quite challenging, especially due to the low processing power available in such kind of vehicles, and most of the so far proposed SLAM algorithms for underwater vehicles can't cope with that. Moreover, the lack of natural features sometimes requires beacons or other artificial features to be deployed on site. One of the first instances of a deployable underwater implementation of the SLAM algorithm was presented by Williams (2001). Similar EKF-based approaches followed as for example the work by Williams and Mahon (2006); Roman and Singh (2005); Koh et al. (2009). Fairfield and Wettergreen (2008) also presented some work using PF-based algorithms for SLAM. Moreover, there has been a significant work on developing SLAM solutions for artificial man-made structured environments. Examples of this are what was presented by Ribas et al. (2008); Burguera et al. (2010). Sometimes the use of artificial features can be quite challen- ging, or even some times not possible. In such situations the use of featureless SLAM methods like Bathymetric SLAM or Scan Matching can be very interesting.

\subsection{Bathymetric SLAM}

In the context of underwater Terrain Based Navigation featureless SLAM approaches become very relevant, as they do not require to explicitly identify or track any features. Instead, such approaches use only raw sensor measurements and thus, making them generally applicable to unstructured environments. Bathymetric SLAM tries to use sonar data measurements to build a surface terrain map, and use this map to bound the vehicle navigational error.

Roman and Singh (2005) first proposed the use of bathymetric data with SLAM methodology, using an EKF to enforce consistency between the vehicle navigation data and the acoustic mapping data. The Bathymetric Particle SLAM (BPSLAM) filter, a variation of the DPSLAM filter, was introduced by Barkby et al. (2009a) as another alternative for a featureless SLAM approach based on bathymetric data. Building up on previous work by Fairfield et al. (2007); Fairfield and Wettergreen (2008), the BPSLAM consists on a RBPF for the estimation step, together with an efficient gridded map representation of the space, that maintains a single estimate of seabed depth in each grid cell, along with its uncertainty. It has been shown that BPSLAM improves navigation and map consistency when compared with navigation methods that do not implement any form of map-based localization. Further improvements and alternative map representations to the original algorithm were developed by Barkby and Williams (2009b); Barkby et al. (2011a), (2011b). Later, Kim and Sang (2011) introduced a computationally efficient SLAM algorithm, able to the elevation changes in undulating terrain and simultaneously localize the vehicle's position relative to the map. Stuckey (2012) proposed an EKFbased Selective Bathymetric SLAM algorithm to improve the navigational accuracy of underwater vehicles.

\subsection{Scan matching}

Scan matching is a technique that estimates the robot's relative displacement between two poses by maximizing the overlap between range scans normally gathered with a laser or sonar sensor. On a purely geometrical perspective, scan matching can be also interpreted as the problem of finding the rotations and translations that maximize the overlapping of two sets of acquired data. Several scan-matching based techniques have been proposed in the literature, both in man-made structured environments and partially structure natural environments, with encouraging results. A rather complete overview in the field was made by Mallios et al. (2014).

Several scan matching algorithms exist, being the most popular variations of the Iterative Closest Point (ICP). However, it should be noted that other approaches exist, like the one proposed by Censi (2006), making use of a Generalized Hough Transform. It has been noted that original ICP approaches don't explicitly provide an uncertainty estimation but several authors tried to address this issue. Researchers from both the Universitat de Girona and from HeriotWatt University have consistently published various articles regarding the problem of SLAM based on Scan Matching techniques. The interested reader should refer for example to the works by Hernández et al. (2009a), (2009b); Zandara et al. (2013); Mallios et al. (2009). Recently, the simultaneous use of two complementary EKFs has been proposed. This novel approach was proposed by Mallios et al. (2010), on which one EKF is responsible to estimate the local robot motion during a scan, so that motion-induced distortions affecting the scans can be compensated. On the other hand, the second $\mathrm{EKF}$ is used to estimate the global trajectory of the vehicle. 


\section{Summary}

In this state-of-the-art survey, different approaches and aspects of bathymetry-based Terrain Based Navigation for underwater vehicles have been covered. We started with an overview of the different navigation methods for AUVs and discussed the general TBN problem. Roughly, there are two distinctive methods to solve the matching problem between maps and measurements: the Gradient based approach, and the Search-area method. Gradient methods use terrain measurements directly as navigation input but require the computation of terrain gradients, which hasn't been considered very interesting. On the other hand, search-area methods have been widely used in the literature, and only the outputs of a correlation-based matching function are used as navigation input.

After that, we described the different sonar sensors that can be used to obtain terrain measurements. and provided an overview on the most significant real-time implementations that can be found on the literature. The state-space models and Bayesian Estimation algorithms were presented. The most successful implementations of underwater TBN have achieved meter-level performance by utilizing high accuracy inertial navigation systems together with high quality sonars and terrain maps. In recent work it was also shown that similar accuracies can be attained even when using lower grade inertial sensors. Initial approaches for underwater TBN were focused on the use of MBE, mostly due to its intrinsic properties like large swath. Nonetheless, numerous studies were also performed where lower accuracy bathymetric sensors, like DVL sensors and even single beam altimeters, were able to deliver fairly accurate results.

While the Terrain Based Navigation estimation problem is partially solved, there are still some challenges that remain to be addressed. Robust estimators able to deal with multi-modality scenarios, when using sonar with a small number of beams, have already been proposed for aerial vehicles, but this is still an open problem for underwater environments. An interesting way to improve TBN accuracy may also consist on finding optimal routes that gather the maximum of information coming from the terrain sensor. Furthermore, a complete framework that deals with the use of features for relative positioning and control, like pipelines and harbour walls, together with terrain profiles can also be of great interest.

Application for underwater TBN are still limited by the need for an a priori map, but when this is not the case concurrent mapping and navigation methods are required. Despite some of the research already presented, the use of SLAM in unstructured environments, such as natural terrains, is still an open question. Finally, TBN algorithms that minimize the use of sonar could also be extremely interesting for many military applications.

\section{Acknowledgement}

This work is financed by the ERDF-European Regional Development Fund through the Operational Programme for Competitiveness and Internationalisation - COMPETE 2020 Programme within project $\ll$ POCI-01-0145-FEDER-006961», and by National Funds through the FCT-Fundação para a Ciência e a Tecnologia (Portuguese Foundation for Science and Technology) as part of project UID/EEA/ 50014/2013. The first author was supported by the Portuguese Foundation for Science and Technology through the Ph.D. grant SFRH/ $\mathrm{BD} / 70727 / 2010$.

\section{References}

Vickery, K., 1998. Acoustic positioning systems. a practical overview of current systems. In: Proceedings of the 1998 Workshop on Autonomous Underwater Vehicles, AUV'98., IEE, Cambridge, USA. pp. 5-17.

Kullander, L., 1989. Development of a terrain navigation system for AUV's. In: Proceedings of the 6th International Symposium on Unmanned Untethered Submersible Technology, IEEE. pp. 494-501.
Alexander, J., Cheng, Y., Zheng, W., Trawny, N., Johnson, A., 2012. A Terrain Relative Navigation sensor enabled by multi-core processing. In: IEEE Aerospace Conference, pp. 1-11.

Anonsen, K.B., Hagen, O.K., 2011. Recent developments in the HUGIN AUV terrain navigation system. In: Proceedings of MTS/IEEE Oceans'11 Conference, Kona, Hawai, USA, pp. 1-7.

Anonsen, K.B., Hallingstad, O., 2006. Terrain aided underwater navigation using point mass and particle filters. In: IEEE/ION Position, Location, and Navigation Symposium, pp. 1027-1035.

Anonsen, K.B., Hallingstad, O., Hagen, O.K., 2007. Bayesian terrain-based underwater navigation using an improved state-space model. In: Symposium on Underwater Technology and Workshop on Scientific Use of Submarine Cables and Related Technologies, pp. 499-505.

Anonsen, K. B. 2010. Advances in Terrain Aided Navigation for Underwater Vehicles (Ph. D. thesis). Norwegian University of Science and Technology, Faculty of Information Technology, Mathematics and Electrical Engineering, Department of Engineering Cybernetics.

Arulampalam, M., Maskell, S., Gordon, N., Clapp, T., 2002. A tutorial on particle filters for online nonlinear/non-gaussian bayesian tracking. IEEE Trans. Signal Process. 50, 174-188.

Aulinas, J., Llado, X., Salvi, J., Petillot, Y.R., 2010. Feature based slam using side-scan salient objects. In: Proceedings of MTS/IEEE Oceans'11 Conference, Seattle, USA, pp. $1-8$.

Aulinas, J., Carreras, M., Llado, X., Salvi, J., Garcia, R., Prados, R., Petillot, Y.R., 2011 Feature extraction for underwater visual SLAM. In: Proceedings of MTS/IEEE Oceans'11 Conference, Santander, Spain, pp. 1-7.

Bachmann, A., Williams, S.B., 2003. Terrain aided underwater navigation - a deeper insight into generic monte carlo localization. In: Proceedings of the Australasian Conference on Robotics \& Automation, December 1-3, Brisbane, Australia, pp. 1-7.

Bahr, A., Leonard, J.J., Fallon, M.F., 2009. Cooperative localization for autonomous underwater vehicles. Int. J. Robot. Res. 28, 714-728.

Barkby, S., Williams, S., Pizarro, O., Jakuba, M., 2009b. Incorporating prior bathymetric maps with distributed particle bathymetric SLAM for improved AUV navigation and mapping. In: Proceedings of MTS/IEEE Oceans'09 Conference, IEEE, Biloxi, USA. pp. 1-7.

Barkby, S., Williams, S., Pizarro, O., Jakuba, M., 2009a. An efficient approach to bathymetric SLAM. In: 2009 IEEE/RSJ International Conference on Intelligent Robots and Systems, pp. 219-224.

Barkby, S., Williams, S.B., Pizarro, O., Jakuba, M.V., 2011a. A featureless approach to efficient bathymetric SLAM using distributed particle mapping. J. Field Robot. 28, 19-39.

Barkby, S., Williams, S.B., Pizarro, O., Jakuba, M.V., 2011b. Bathymetric SLAM with no map overlap using Gaussian Processes. In: IEEE/RSJ International Conference on Intelligent Robots and Systems, pp. 1242-1248.

Bergem, O.r., 1993. Bathymetric Navigation of Autonomous Underwater Vehicles using a Multibeam Sonar and a Kalman Filter with Relative Measurent Covariance Matrices. (Ph.D. thesis). University of Trondheim, College of Arts and Science, Department of Informatics and Computer Science.

Bergman, N., Ljung, L., Gustafsson, F., 1999. Terrain navigation using Bayesian statistics. Control Syst. IEEE 19, 33-40.

Bergman, N., 1997. On the Cramer-Rao bound for terrain-aided navigation. Technical Report. Dept of EE, Linköping University, Linköping.

Bergman, N., 1999. Recursive Bayesian Estimation-Navigation and Tracking Applications (Ph.D. thesis). Department of Electrical Engineering, Linkoping University, Sweden.

Burguera, A., Oliver, G., 2016. High-resolution underwater mapping using side-scan sonar. PLOS One 11, 1-41.

Burguera, A., Oliver, G., Gonzalez, Y., 2010. Scan-based SLAM with trajectory correction in underwater environments. In: 2010 IEEE/RSJ International Conference on Intelligent Robots and Systems (IROS), pp. 2546-2551.

Carlström, J., 2007. Results from sea trials of the swedish auv62f's terrain navigation system. In: Proceedings of the 15th International Symposium on Unmanned Untethered Submersible Technology (UUST'07), Durham, NH, USA, pp. 250-300.

Carreno, S., Wilson, P., Ridao, P., Petillot, Y., 2010. A survey on terrain based navigation for AUVs. In: Proceedings of MTS/IEEE Oceans'10 Conference, Seattle, USA, pp. 1-7.

Censi, A., 2006. Scan matching in a probabilistic framework. In: Proceedings 2006 IEEE International Conference on Robotics and Automation ICRA, 2006, pp. 2291-2296.

Chen, Z., 2003. Bayesian filtering: from Kalman filters to particle filters, and beyond. Technical Report. McMaster University.

Claus, B., Bachmayer, R., 2015. Terrain-aided navigation for an underwater glider. J. Field Robot. 32, 935-951.

Cowie, M., Wilkinson, N., Powlesland, R., 2008. Latest development of the terprom digital terrain system (dts). In: 2008 IEEE/ION Position, Location and Navigation Symposium, pp. 1219-1229.

Csorba, M., 1997. Simultaneous Localisation and Mapping. Phd. University of Oxford.

Dektor, S., Rock, S., 2012. Improving robustness of terrain-relative navigation for AUVs in regions with flat terrain. In: Autonomous Underwater Vehicles (AUV), IEEE/OES, pp. 1-7.

Dektor, S., Rock, S., 2014. Robust adaptive terrain-relative navigation. In: Proceedings of MTS/IEEE Oceans'14 Conference, St. John's, Canada, pp. 1-10.

Donovan, G.T., 2011. Development and testing of a real-time terrain navigation method for AUVs. In: Proceedings of MTS/IEEE Oceans'11 Conference, Kona, Hawai, USA, pp. $1-9$.

Donovan, G.T., 2012. Position error correction for an autonomous underwater vehicle inertial navigation system (ins) using a particle filter. IEEE J. Ocean. Eng. 37, 431-445. 
Eliazar, A.I., Parr, R, 2003. DP-SLAM: Fast, robust simultaneous localization and mapping without predetermined landmarks. In: IJCAI, pp. 1135-1142.

Eliazar, A.I., Parr, R., 2004. DP-SLAM 2.0. In: Proceedings of the IEEE International Conference on Robotics and Automation, ICRA '04. vol. 2, pp. 1314-1320.

Enns, R., Morrell, D., 1995. Terrain-aided navigation using the Viterbi algorithm. J Guid. Control Dyn. 18, 1444-1449.

Fairfield, N., Wettergreen, D., 2008. Active localization on the ocean floor with multibeam sonar. In: Proceedings of MTS/IEEE Oceans'08 Conference, Quebec City, Canada. pp. 1-10.

Fairfield, N., Kantor, G., Wettergreen, D., 2007. Real-time SLAM with octree evidence grids for exploration in underwater tunnels. J. Field Robot. 24, 3-21.

Fairfield, N., Wettergreen, D., Kantor, G., 2010. Segmented SLAM in three-dimensional environments. J. Field Robot. 27, 85-103.

Fallon, M.F., Papadopoulos, G., Leonard, J.J., 2010. A measurement distribution framework for cooperative navigation using multiple AUVs. In: IEEE International Conference on Robotics and Automation (ICRA), pp. 4256-4263.

Ferreira, B., Matos, A., Cruz, N., 2010. Single beacon navigation: Localization and control of the mares AUV. In: Proceedings of MTS/IEEE Oceans'10 Conference, IE, Seattle, USA, pp. 1-9.

Fofonoff, N., R.C.Millard, 1983. Algorithms for computation of fundamental properties of seawater. Technical Report. UNESCO.

Groves, P.D., 2013. Principles of GNSS, Inertial, and Multisensor Integrated Navigation Systems. GNSS/GPS, Artech House, Second edition.

Hagen, P.E., Øyvind Hegrenaes, Jalving, B., Øivind Midtgaard, Wiig, M., Hagen, O.K., 2009. Making AUVs truly autonomous. In: (Ed.), A.V.I. (Ed.), Underwater Vehicles, InTech, pp. 129-152.

Hagen, O.K., Anonsen, K.B., Saebo, T.O., 2011. Low altitude AUV terrain navigation using an interferometric sidescan sonar. In: Proceedings of MTS/IEEE Oceans'11 Conference, Kona, Hawai, USA, pp. 1-8.

Hagen, O.K., 2008. From pressure to depth: estimation of underwater vertical position. Technical Report. FFI, Avd Maritime Systemer.

Hansen, R.E., Saebo, T.O., Gade, K., Chapman, S., 2003. Signal processing for AUV based interferometric synthetic aperture sonar. In: Proceedings of MTS/IEEE Oceans'03 Conference, San Diego, CA, USA, pp. 2438-2444.

Hansen, R.E., Callow, H.J., Sabo, T.O., Synnes, S.A.V., 2011. Challenges in seafloor imaging and mapping with synthetic aperture sonar. IEEE Trans. on Geosci. Remote Sens. 49, 3677-3687.

Hansen, R.E., 2011. Introduction to synthetic aperture sonar. In: Kolev, P.N. (Ed.), Sonar Systems. InTech, pp. 3-28.

Hausler, A.J., Saccon, A., Pascoal, A.M., Hauser, J., Aguiar, A.P., 2013. Cooperative AUV motion planning using terrain information. In: Proceedings of MTS/IEEE Oceans'13 Conference, Bergen, Norway, pp. 1-10.

Hernández, E., Ridao, P., Ribas, D., Batlle, J., 2009a. MSISpIC: A probabilistic scan matching algorithm using a mechanical scanned imaging sonar. J. Phys. Agents 3, 1-9.

Hernández, E., Ridao, P., Ribas, D., Mallios, A., 2009b. Probabilistic sonar scan matching for an AUV. In: IEEE/RSJ International Conference on Intelligent Robots and Systems, IROS, pp. 255-260.

Hollowell, J., 1990. Heli/SITAN: a terrain referenced navigation algorithm for helicopters. In: IEEE PLANS '90 Position Location and Navigation Symposium, Record. The 1990's - A Decade of Excellence in the Navigation Sciences, pp. 616625.

Hostetler, L., Andreas, R., 1983. Nonlinear Kalman filtering techniques for terrain-aided navigation. IEEE Trans. Autom. Control 28, 315-323.

Hostetler, L.D., 1978. Optimal terrain-aided navigation systems. In: Guidance and Control Conference, Guidance, Navigation, and Control and Co-located Conferences, Palo Alto, California, USA, pp. 20-30.

Houts, S.E., Rock, S.M., McEwen, R., 2012. Aggressive terrain following for motionconstrained AUVs. In: IEEE/OES Autonomous Underwater Vehicles (AUV), pp. 1-7.

Jalving, B., Mandt, M., Hagen, O.K., Pøhner, F., 2004. Terrain referenced navigation of auvs and submarines using multibeam echo sounders. In: Proceedings of the UDT Europe 2004, Nice, France. pp. 1-10.

Jian, S., Jing-yuan, Z., Feng, Z., 2012. Alterable resolution point-mass filter algorithm for underwater terrain matching method. Journal of Chinese Inertial Technology.

Johnson, A.E., Montgomery, J.F., 2008. Overview of terrain relative navigation approaches for precise lunar landing. In: IEEE Aerospace Conference, pp. 1-10.

Karabork, A., 2010. Terrain aided navigation. Master's thesis. The Graduate School of Natural and Applied Sciences of Middle East Technical University.

Karlsson, R., Gusfafsson, F., Karlsson, T., 2003. Particle filtering and Cramer-Rao lower bound for underwater navigation. In: Proceedings IEEE International Conference on Acoustics, Speech, and Signal Processing (ICASSP '03), pp. VI-65-8.

Kedong, W., Lei, Y., Wei, D., Junhong, Z., 2006. Research on iterative closest contour point for underwater terrain-aided navigation. In: Yeung, D.Y., Kwok, J., Fred, A., Roli, F., de Ridder, D. (Eds.), Structural, Syntactic, and Statistical Pattern Recognition. Springer Berlin Heidelberg. volume 4109 of Lecture Notes in Computer Science, pp. 252-260.

Kedong, W., 2010. Influence of application conditions on terrain-aided navigation. In: Proceedings of the IEEE 8th World Congress on Intelligent Control and Automation, pp. 391-396.

Kim, T., Kim, J., 2014. Nonlinear filtering for terrain-referenced underwater navigation with an acoustic altimeter. In: Proceedings of MTS/IEEE Oceans'14 Conference, Taipei, Taiwan, pp. 1-6.

Kim, J., Sang, H., 2011. An approach towards online Bathymetric SLAM. In: Proceedings of MTS/IEEE Oceans'11 Conference, IE, Kona, Hawai, USA. pp. 1-6.

Kinsey, J.C., Eustice, R., Whitcomb, L.L., 2006. A survey of underwater vehicle navigation: recent advances and new challenges. In: Proceedings of the 7 th
Conference on Manoeuvring and Control of Marine Craft (MCMC'2006), pp. 1-12.

Kjetil, A., 2007. Sigma point Kalman filter for underwater terrain-based navigation. In Sauro, L. (Ed.), In: Proceedings of the IFAC Conference on Control Applications in Marine Systems, Bol, Croatia, September, pp. 106-110.

Koh, A.C.T., Wijesoma, W.S., Pua, S.L., Lee, K.W., Kalyan, B., Avenue, N., 2009. Shallow Waters SLAM Experiments on Meredith AUV using forward looking sonar. In: Proceedings of MTS/IEEE Oceans'09 Conference, Biloxi, USA, pp. 1-6.

LaPointe, C.E.G., 2006. Virtual Long Baseline (VLBL) autonomous underwater vehicle navigation using a single transponder (Master's thesis). Massachusetts Institute of Technology.

Larsen, M., 2000. Synthetic long baseline navigation of underwater vehicles. In: Proceedings of MTS/IEEE Oceans'00 Conference, IEE, Providence, USA., vol. 3, pp. 2043-2050.

Leonard, J.J., Bennett, A.A., Smith, C.M., Feder, H.J.S., 1998. Autonomous underwater vehicle navigation. In: MIT Marine Robotics Laboratory Technical Memorandum. Dept. of Ocean Engineering, Massachussets Institute of Technology, Cambridge, MA, USA. pp. 1-17.

Lucido, L., Pesquet-Popescu, B., Opderbecke, J., Rigaud, V., Deriche, R., Zhang, Z Costa, P., Larzabal, P., 1998. Segmentation of bathymetric profiles and terrain matching for underwater vehicle navigation. Int. J. Syst. Sci. 29, 1157-1176.

Lucido, L., Opderbecke, J., Rigaud, V., Deriche, R., Zhang, Z., 2004. A terrain referenced underwater positioning using sonar bathymetric profiles and multiscale analysis. In: Proceedings of MTS/IEEE Oceans'96 Conference, Fort Lauderdale, USA, pp. 417421.

Makumder, S., 2001. Sensor Fusion and Feature Based Navigation for Subsea Robots (Ph.D. thesis). The University of Sidney.

Mallios, A., Ridao, P., Hernández, E., Davi, Maurelli, F., Petillot, Y., 2009. Pose-based SLAM with probabilistic scan matching algorithm using a mechanical scanned imaging sonar. In: Proceedings of MTS/IEEE Oceans'09 Conference, Bremen, Germany, pp. 1-6.

Mallios, A., Ridao, P., Ribas, D., Maurelli, F., Petillot, Y., 2010. EKF-SLAM for AUV navigation under probabilistic sonar scan-matching. In: IEEE/RS.J International Conference on Intelligent Robots and Systems (IROS) , pp. 4404-4411.

Mallios, A., Ridao, P., Ribas, D., Hernández, E., 2014. Scan matching slam in underwater environments. Auton. Robots 36, 181-198.

Manual on Hydrography, 2011. Manual on Hydrography. International Hydrographic Bureau. 4, Quai Antoine 1er B.P. 445-MC 98011 MONACO Cedex Principauté de Monaco.c-13 edition.

Massa, D.D., Stewart, W., 1997. Terrain-relative navigation for autonomous underwate vehicles. In: Proceedings of MTS/IEEE Oceans'97 Conference, Halifax, Canada, pp. 541-546.

Massa, D.D., 1997. Terrain-Relative Navigation for Autonomous Underwater Vehicles (Ph.D. thesis). Massachusetts Institute of Technology/Woods Hole Oceanographic Institution.

Meduna, D.K., Rock, S.M., McEwen, R.S., 2008. Low-cost terrain relative navigation for long-range AUVs. In: Proceedings of MTS/IEEE Oceans'08 Conference, IEE, Quebec City, Canada, pp. 1-7.

Meduna, D.K., Rock, S.M., McEwen, R.S., 2010. Closed-loop terrain relative navigation for AUVs with non-inertial grade navigation sensors. In: IEEE/OES Autonomous Underwater Vehicles, pp. 1-8.

Meduna, D.K., 2011. Terrain Relative Navigation for Sensor-Limited Systems with Application to Underwater Vehicles. Ph.D. Standord University.

Melo, J., Matos, A., 2013. On the use of particle filters for terrain based navigation of sensor-limited AUVs. In: Proceedings of MTS/IEEE Oceans'13 Conference, IEE, Bergen, Norway. pp. 1-8.

Montemerlo, M., Thrun, S., Koller, D., Wegbreit, B., 2002. Fastslam: A factored solution to the simultaneous localization and mapping problem. In: Proceedings of the Eighteenth National Conference on Artificial Intelligence. American Association for Artificial Intelligence, Menlo Park, CA, USA. pp. 593-598.

Morice, C., Veres, S., McPhail, S., 2009. Terrain referencing for autonomous navigation of underwater vehicles. In: Proceedings of MTS/IEEE Oceans'09 Conference, Bremen, Germany, pp. 1-7.

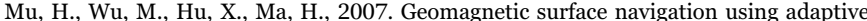
EKF. In: Proceedings of the 2nd IEEE Conference on Industrial Electronics and Applications, pp. 2821-2825.

Multibeam Sonar Theory of Operation, 2000. Multibeam sonar theory of operation. L-3 communications SeaBeam instruments. 141Washington Street East Walpole, MA $02032-1155$.

Murangira, A., Musso, C., Dahia, K., Allard, J., 2011. Robust regularized particle filter for terrain navigation. In: Proceedings of the 14th International Conference on Information Fusion (FUSION), pp. 1-8.

Nakatani, T., Ura, T., Sakamaki, T., Kojima, J., 2009. Terrain based localization for pinpoint observation of deep seafloors. In: Proceedings of MTS/IEEE Oceans'09 Conference, Bremen, Germany, pp. 1-6.

Nordlund, P.J., Gustafsson, F., 2009. Marginalized particle filter for accurate and reliable terrain-aided navigation. IEEE Trans. Aerosp. Electron. Syst. 45, 1385-1399.

Nygren, I., Jansson, M., 2004. Terrain navigation for underwater vehicles using the correlator method. IEEE J. Ocean. Eng. 29, 906-915.

Nygren, I., 2005. Terrain navigation for underwater vehicles (Ph.D. thesis). Royal Institute of Technology (KTH), School of Electrical Engineering.

Nygren, I., 2008. Robust and efficient terrain navigation of underwater vehicles. In: IEEE/ION Position, Location and Navigation Symposium, pp. 923-932.

Padial, J., Dektor, S., Rock, S.M., 2014a. Correlation of imaging sonar acoustic shadows and bathymetry for ROV terrain-relative localization. In: Proceedings of MTS/IEEE Oceans'14 Conference, IE, Taipei, Taiwan. pp. 1-10.

Padial, J., Dektor, S., Rock, S.M., 2014b. Measurement model development for the 
correlation of imaging sonar acoustic shadows and bathymetry for rov terrainrelative localization. In: Proceedings of the MTS/IEEE Oceans'14 Conference, St. John's, Canada, pp. 1-9.

Paull, L., Saeedi, S., Seto, M., Li, H., 2014. AUV navigation and localization: a review. IEEE J. Ocean. Eng. 39, 131-149.

Ren, Z., Chen, L., Zhang, H., Wu, M., 2008. Research on geomagnetic-matching localization algorithm for unmanned underwater vehicles. In: IEEE ICIA International Conference on Information and Automation, pp. 1025-1029.

Ribas, D., Ridao, P., Tardós, J.D., Neira, J., 2008. Underwater slam in man-made structured environments. J. Field Robot. 25, 898-921.

Roman, C., Singh, H., 2005. Improved vehicle based multibeam bathymetry using submaps and SLAM. In: 2005 IEEE/RSJ International Conference on Intelligent Robots and Systems, pp. 3662-3669.

Schon, T.B., Karlsson, R., Gustafsson, F., 2006. The marginalized particle filter in practice. In: IEEE Aerospace Conference, pp. 1-11.

Stalder, S., Bleuler, H., Ura, T., 2008. Terrain-based navigation for underwater vehicles using side scan sonar images. In: Proceedings of MTS/IEEE Oceans'08 Conference, Quebec CIty, Canada, pp. 1-3.

Strauss, O., Comby, F., Aldon, M.J., 1999. Multibeam sonar image matching for terrainbased underwater navigation. In: Proceedings of MTS/IEEE Oceans'99 Conference, Seattle, USA, pp. 882-887.

Stuckey, R.A., 2012. Navigational error reduction of underwater vehicles with selective Bathymetric SLAM. In: IFAC Workshop on Navigation, Guidance and Control of Underwater Vehicles (NGCUV'2012), Porto, pp. 1-8.

Stutters, L., Tiltman, C., Brown, D.J., 2008. Navigation technologies for autonomous underwater vehicles. IEEE Trans. Syst. Man Cybern. Part C. (Appl. Rev.) 38, 581-589.

Teixeira, F.C., Pascoal, A., Maurya, P., 2012. A novel particle filter formulation with application to terrain-aided navigation. In: Proceedings of the 3rd \{IFAC $\}$ Workshop on Navigation, Guidance and Control of Underwater Vehicles, volumes 45, 132-139.

Thrun, S., Burgard, W., Fox, D., 2005. Probabilistic Robotics (Intelligent Robotics and Autonomous Agents Series). The MIT Press.

Vaman, D., 2012. TRN history, trends and the unused potential. In: IEEE/AIAA 31st
Digital Avionics Systems Conference (DASC), pp. 1A3-1-1A3-16.

Wang, S., Zhang, H., Yang, K., Tian, C., 2010. Study on the underwater geomagnetic navigation based on the integration of TERCOM and $\mathrm{K}$-means clustering algorithm. In: Proceedings of MTS/IEEE Oceans'10 Conference, Sidney, Australia, pp. 1-4.

Wilby, A.D., 1999. The advantages, challenges and practical implementation of an interferometric swath bathymetry system. In: Proceedings of MTS/IEEE Oceans' 99 Conference, Seattle, USA, vol. 1, pp. 23-29.

Williams, S., Mahon, I., 2006. A terrain-aided tracking algorithm for marine systems. In: Yuta, S., Asama, H., Prassler, E., Tsubouchi, T., Thrun, S. (Eds.), Field and Service Robotics: Recent Advances in Reserch and Applications. Springer Berlin Heidelberg, Berlin, Heidelberg, pp. 93-102.

Williams, S.B., 2001. Efficient Solutions to Autonomous Mapping and Navigation Problems. Ph.D. The University of Sidney.

Willumsen, A.B., Hagen, O.K., Boge, P.N., 2007. Filtering depth measurements in underwater vehicles for improved seabed imaging. In: Proceedings of MTS/IEEE Oceans'07 Conference, IEE, Aberdeen, Scotland. pp. 1-6.

Woock, P., Frey, C., 2010. Deep-sea AUV navigation using side-scan sonar images and SLAM. In: Proceedings of MTS/IEEE Oceans'10 Conference, IE, Sidney, Australia. pp. $1-8$.

Woock, P., 2012. Survey on suiTable 3D features for sonar-based underwater navigation. In: Proceedings of MTS/IEEE Oceans'12 Conference, Yeosu, Korea, pp. 1-6.

Yuan, G., Zhang, H., Yuan, K., Zhu, L., 2012. Improved SITAN algorithm in the application of aided inertial navigation. In: International Conference on Measurement, Information and Control (MIC), pp. 922-926.

Zandara, S., Ridao, P., Ribas, D., Mallios, A., Palomer, A., 2013. Probabilistic surface matching for bathymetry based SLAM. In: IEEE International Conference on Robotics and Automation (ICRA), pp. 40-45.

Zerr, B., Mailfert, G., Bertholom, A., Ayreault, H., 2005. Sidescan sonar image processing for AUV navigation. In: Proceedings of MTS/IEEE Oceans'05 Conference, Brest, France, vol. 1, pp. 124-130.

Zhao, J., Wang, S., Wang, A., 2009. Study on underwater navigation system based on geomagnetic match technique. In: Proceedings of the IEEE 9th International Conference on Electronic Measurement \& Instruments, pp. 3-259. 